\title{
The System of Free Access Galactica to Compute Interactions of N-Bodies
}

\author{
Joseph J. Smulsky \\ Institute of Earth Cryosphere SB RAS, 625000, Tumen, P.O.Box 1230, Russia \\ Email: jsmulsky@mail.ru
}

\begin{abstract}
The Galactica System is intended for resolving N-body interaction problems. It is based on a high-precision method for solving differential equations of motion of bodies whose interaction forces are inversely proportional to the squared distance. The differential equations of motion and their solution method are given in paper. The structure of the input file, the files of initial conditions and output files are described. The examples of these files for different solved tasks are presented. The actions of executable files are explained. The paper describes all the theoretical and practical issues so that even a novice researcher could use Galactica System in his/her works.
\end{abstract}

Index Terms - Gravitational and Coulomb Interaction, Equations of Motion, Computer Calculations, Solar System, Free Access

\section{INTRODUCTION}

Galactica is the software that was created in order to study the evolution of the Solar System in geological ages. It is based on the high-precision method of integrating differential equations of motion of material points while they interact according to the Newton law of gravitation. Galactica was used to solve several problems [1], including: the evolution of the Solar System for 100 million years, the optimal flight of a spacecraft to the Sun the evolution of motion of Apophis and 1950DA asteroids and converting their orbits into orbits of satellites of the Earth; the Earth's axis evolution simulation, the effect of the Sun's rotation on the planetary orbits, etc.

Since the program Galactica and the accumulated set of additional tools can solve various problems of gravitational interaction of bodies, the free access Galactica System was developed. In addition to the program, the Galactica System includes additional components for problem setting, leading it to solution, the solution of the problem and the analysis of results. The system is available at: http://www.ikz.ru/ smulski/GalactcW/. This paper presents the User Manual for the system.

The Galactica methods differ much [1] from methods currently used in the celestial and space dynamics. The result of this difference is higher precision of solutions and a wider and more diverse range of problems to set and solve. For instance, although the method is based on the interaction of material points, Galactica solved the problem of the Earth's rotation evolution and the influence of the Sun's rotation. These problems no longer consider the Earth and the Sun as material points.

The program Galactica resolves issues of highaccuracy integration of differential equations and the information output of the computation process and its accuracy. The system Galactica, particularly in this Paper, pays a lot of attention to the problem statement. We hope they will help researchers to expand the scope of problems to be solved using Galactica.

Those who understand theoretical mechanics believe it is a very simple science. Unfortunately, numerous branches in mechanics in the $20^{\text {th }}$ century resulted in various interpretations of mechanics foundation. So, in the Paper we included the basic principles, which are needed for solving the problems.

Galactica solves equations in dimensionless form. On the one hand, this optimizes the computations, whereas on the other hand, the same solution can be applied to the problems of various scales. For instance, the solutions of the planetary system formation and its evolution can be applied to the galaxy formation and its evolution. To do this, the Manual contains the formulas to convert dimensionless values into dimensional ones.

The task has to go through several stages on the way from the initial idea to the final result. Their solution requires a lot of different knowledge. And it is not every researcher who has it. To facilitate the researcher's job, the Manual contains the description of most of the stages, including examples of studies. One of the important stages is the analysis of results. Further on, we will add this paragraph to the Manual and attach the programs to analyze the trajectories.

Galactica is available at the address above to work at a personal computer. To solve larger scale problems, Galactica is created at the supercomputer at the Siberian Supercomputer Center (SSCC), the Siberian Branch of the Russian Academy of Science, in Novosibirsk. One of the Galactica versions was developed in MPI environment to run parallel computations. However opportunities of SSCC are limited, therefore the free access on the supercomputer till now is not authorized.

Since the Coulomb interaction is similar to the Newtonian one, we have also developed a Galactica version for computing interactions of charged particles. Since the early $20^{\text {th }}$ century, the method of statistical 
physics has prevailed in micro-world to study the behavior of ensembles of particles. This was supported by the fact that at that time, it was impossible to solve the problems of deterministic interaction of many charged particles. Now, the theoretic problems have been solved [2], [3] and they help to set the initial conditions for atomic shells. Hence, we expect that Galactica for the Coulomb interaction will provide a more deterministic vision on the microcosm. As soon as at least one problem in this sphere is solved, we will add the Coulomb interaction to Galactica.

The Galactica System is designed as an open system. We expect that as other researchers solve more and more problems, they will save their files with initial conditions, computations, and other work results in the relevant folders. On the one hand, they will help other researchers to reproduce the solutions. If the result is the same, then a second researcher will validate the conclusions made by a first one. On the other hand, other researchers may set and solve new problems using the previous researchers' results.

The main content of the paper is organized such way. The Essentials of Galactica are in the saecond section. The differential equations of motion for material-point bodies and their solution method are described in sections 3 and 4, accordingly. The description of input file galacf26.dat is in section 5 . Here there are parameters for general description, for functions of interaction region, for output of trajectories, for on-screen display and others. The files of initial conditions and integration data are described in section 6 . The example of MathCad program for generating an initial condition file is in Appendix 1. In sections 7 and 8 there are descriptions of files of trajectories 1t, 2t... traekt.prn and close-approach file dice.dat. On-screen representation of integration data is shown in section 9. The modes of display in the standard FORTRAN graphics are showed in Appendix 2. In Sections 10, 11 and 12 there are examples of input files, of initial condition files and of executable files. How to generate the initial condition file are explained in section 13. The sequence of the problem solution is submitted in section 14.

\section{ESSENTIALS OF GALACTICA}

Galactica is a system intended for solving problems in mechanical interaction of material-point bodies. The interaction is assumed Newtonian. Galactica files are located in folder GalactcW. This folder contains other folders, InCndFls, InpFiles and RunFiles, and also files GalDiscrp.pdf and GalDiscrE.pdf that contain respectively a Russian and English description of Galactica. The zipped folders can be downloaded at http://www.ikz.ru/ smulski/GalactcW/GalacFoldrs.zip.

The system includes named executable files, for instance, glc3b30, to be run on a supercomputer under Unix OS, or glc3b30.exe, to be run under DOS; an input file galacf26.dat, and a named initial data file specifying the interacting bodies and the initial conditions of their motion, for instance, sn69JPA2.dat. Below, the latter file will be referred to as the initial condition file.
The results yielded by an exe-file are produced as output files. The representation form is defined by keys specified in the input file galacf26.dat. Following a certain number of steps, characteristics of body motions can be issued; files containing such data are named 1,2 , 3... garez28.dat. The file garez28.dat is always produced on completion of the program. This file contains characteristics of bodies at the final calculation step. This is the main form of problem solution output.

Besides, if certain keys are set in the input file galacf26.dat, then parameters defining the trajectory of bodies whose numbers are also defined in the input file are issued, too. The names of the trajectory files are as follows: $1 \mathrm{t}, 2 \mathrm{t}, 3 \mathrm{t}$... traekt.prn. The file size is defined by the volume of the data set in the input file.

If certain keys are set, then a file dice.dat is issued. The file contains data on the time of close approach of bodies, minimal separation between bodies, and the number of a body approached by the given body. Each line in the file dice.dat is formed on accomplishment of a certain number of integration steps. The number of such steps is specified in the file galacf26.dat.

With a certain key set, a binary file (or, depending on the particular computer, an unformatted file) named galgon4 is issued. This file contains the state of the problem in binary form at the last calculation step. On subsequent start of the program, calculation is continued starting from this step, the data contained in the input file and in the initial condition file not being read out. The latter procedure is required in solving problems with long running times. The interruptions in calculations do not worsen the solution accuracy.

In the case of an emergency situation, an error file ErrGlctca is generated; this file contained a description of the error having occurred. Such errors can be encountered in opening files and their reading, and they also can be a result of insufficient array dimensions or impossibility to meet accuracy requirements in calculation of bodies merging together on collision.

In Galactica, the possibility to produce a screen of data is provided. Those parameters common to all bodies allows one to monitor both the execution of the program and the calculation accuracy. In solving a problem on a supercomputer, such data are saved in an output stream file.

In a version of Galactica with PC display graphics, along with common problem parameters, images of bodies with their velocity vectors can be produced. The form of images is defined by keys and parameters set in the input file galacf26.dat.

\section{DIFFERENTIAL EQUATIONS OF MOTION FOR MATERIAL-POINT BODIES}

In modern mechanics, a lot of extraneous features have been accumulated masking the clear idea of how interactions are to be calculated. Clarifications 
concerning the algorithm adopted in Galactica can be found in [4-6].

All quantities in the initial condition and integration data files are used in normalized form. The normalized values results from the reduction of differential equations to non-dimensional form. Consider the equations in more detail.

According to the gravity law, a body with number $k$ attracts a body with number $i$, the attractive force being

$$
\vec{F}_{i k}=-G \frac{m_{i} m_{k}}{r_{i k}^{3}} \vec{r}_{i k}
$$

where $G$ is the constant of gravitation, and $\vec{r}_{i k}$ is the radius-vector that connects the massive body $m_{k}$ with the massive body $m_{i}$.

If the total number of bodies is $n$, then the $i$-th body will be acted upon by all other bodies, with the net interaction force being

$$
\vec{F}_{i}=-G m_{i} \sum_{k \neq i}^{n} \frac{m_{k} \vec{r}_{i k}}{r_{i k}^{3}}
$$

The second law of mechanics, $\vec{a}=\vec{F} / m$, states that, under the action of force (2), the $i$-th body will set in motion relative to an inertial (non-accelerated) reference system with acceleration

$$
\frac{d^{2} \vec{r}_{i}}{d t^{2}}=-G \sum_{k \neq i}^{n} \frac{m_{k} \vec{r}_{i k}}{r_{i k}^{3}}, \quad i=1,2, \ldots, n,
$$

where $\vec{r}_{i}$ is the radius-vector of the body $m_{i}$ relative to some center in the inertial frame of reference. For instance, in treating the interactions between solar-system objects $\vec{r}_{i}$ is the radius-vector of the body $m_{i}$ with respect to the center of mass of the Solar system.

Equation (3) gives a system of $3 n$ nonlinear secondorder differential equations, $n$ being an arbitrary number. The total number of bodies $n$ is specified in the input file galacf26.dat. Besides, the arrays in the program should have dimensions not less than $n$. For instance, the exe-file glc3b30 enables calculation of problems involving up to $n=30$ bodies.

Differential equation (3) is written in a non-accelerated (inertial) frame. However, all bodies move with acceleration, and only their center of mass $C$ in the system of interacting bodies is non-accelerated (on the condition that the action of other bodies on the system of interest can be neglected). As a rule, observations are being made to measure the coordinates and velocities relative to some other body used as a frame. For instance, in the Solar system the coordinates of a massive body $m_{i}$ can be reckoned from the Sun $(\mathrm{S})$; such coordinates are denoted as $x_{S i}, y_{S i}, z_{S i}$, and velocities, $v_{x S i}, v_{y S i}, v_{z S i}$. The latter coordinates and velocities can be used to calculate the coordinates and velocities of the center of mass of the
Solar system, which, in projection onto the axis $x$, have the form

$X_{C}=\sum_{i=1}^{n} m_{i} \cdot x_{S i} / M_{S s} ; \quad V_{x C}=\sum_{i=1}^{n} m_{i} \cdot v_{x S i} / M_{S s}$.

Here, $n$ is the total number of bodies in the Solar system, and $M_{S s}=\sum_{i=1}^{n} m_{i}$ is the mass of the Solar system or, generally, the mass of all interacting bodies.

Note that the motion of a selected body, say, the Sun, varies in time, whereas the reference frame keeps moving without acceleration. That is why the frame relates to a certain time in the motion of the chosen body. For instance, the frame attached to the Earth's equatorial plane refers to the beginning of a 50-year period: system of 1900.0, system of 1950.0, or system of 2000.0.

The coordinates and velocities of bodies relative to the center of mass $C$ taken in projection onto the axis $x$ are

$x_{C i}=x_{S i}-X_{C} ; \quad v_{x C i}=v_{x S i}-V_{x c}$.

Here, the coordinates are expressed in meters; time, in seconds; and masses, in kilograms.

With variables normalized by a characteristic size of the system of interacting bodies $A m$ and their mass $M_{S s}$, equations (3) acquire non-dimensional form [4-5]. For the Solar system, we define the quantity $A m$ so that to render the non-dimensional time $T$ expressed in sidereal centuries. Here, the quantity Am turns out to be close to the semi-axis of the Pluto orbit. In non-dimensional form, the equation projected onto the axis $x$ is

$$
\frac{d v_{x i}}{d T}=-\sum_{k \neq i}^{n} \frac{m_{o k}\left(x_{i}-x_{k}\right)}{R_{i k}^{3}} .
$$

Here, $x_{i}=x_{C i} / A m$ is the non-dimensional mass of the $i$-th body; $m_{o k}=m_{k} / M_{S s}$ is the non-dimensional mass of the $k$-th body; $R_{i k}=\sqrt{\left(x_{i}-x_{k}\right)^{2}+\left(y_{i}-y_{k}\right)^{2}+\left(z_{i}-z_{k}\right)^{2}}$ is the non-dimensional separation between the $i$-th and $k$-th body; $v_{x i}=v_{x C i} \cdot k_{v}$ is the non-dimensional velocity of the $i$ th body; $k_{v}=\sqrt{\frac{A m}{G \cdot M_{S s}}}$ is the velocity coefficient in $\mathrm{sec} / \mathrm{m} ; \quad T=t \cdot k_{t}$ is the non-dimensional time; and $k_{t}=\sqrt{\frac{G \cdot M_{S s}}{A m^{3}}}$ is the time coefficient in sidereal centuries per sec.

In initial condition files, in solar-system problems the non-dimensional time $T=t \cdot k_{t}$ is expressed in sidereal centuries, each century comprising 36525.636042 days.

All quantities in Galactica are used as non-dimensional quantities, including the radii of bodies $\left(R a_{o}\right)$, their momentum $\left(P_{o x}\right)$, angular momentum $\left(M_{o x}\right)$, kinetic $\left(E_{o}\right)$ and thermal $\left(E_{t o}\right)$ energies. Given two scales $A m$ and $M_{S s}$, the coefficients $k_{v}$ and $k_{t}$ can be found. Afterwards, the solution can be rendered dimensional using the formulas 
$t=T / k_{t} ; \quad x_{m i}=x_{i} \cdot A m ; \quad v_{m i}=v_{i} / k_{v} ; \quad R a_{i}=R a_{o i} \cdot A m ;$

$P_{x}=P_{o x} \cdot M_{S s} / k_{v} ; \quad M_{x}=M_{o x} \cdot M_{S s} \cdot A m / k_{v} ;$

$E=E_{o} \cdot M_{S s} / k_{v}^{2} ; E_{t}=E_{t o} \cdot M_{S s} / k_{v}^{2}$.

If the primary scale is the time $T$ expressed, say, in sidereal centuries, then the scale length $A m$ entering the expression for $k_{t}$ can be calculated as

$A m=\left(G \cdot M_{S s} / k_{t}^{2}\right)^{1 / 3}$.

In mode $\mathrm{Kl} 1=0$, i.e. in operation with input data and initial conditions specified for a distributed region filled with substance, a density of bodies $\rho_{b}$ expressed in $\mathrm{kg} / \mathrm{m}^{3}$ is used. Given the scale $A m$, from non-dimensional radius $R a_{o i}$ and mass $m_{o i}$ of bodies one can calculate dimensional quantities:

$R a_{i}=R a_{o} \cdot A m ; \quad m_{i}=m_{o i} \cdot 3 \cdot \pi R a_{i}^{3} \cdot \rho_{b} / 4$.

Then, the total mass of bodies $M_{S s}=\sum_{i=1}^{n} m_{i}$ and the coefficients $k_{v}$ and $k_{t}$ can be calculated. Further calculations are to be carried out by formulas (7).

Thus, the solution produced by Galactica can be recalculated, using scaling factors $A m$ and $M_{S s}$, to various situations. For instance, the problem on evolution of substance ending in the formation of a star and planets can be employed for stars with a widely ranging distribution of substance. The data obtained in solving this problem can also be recalculated to scales over which galaxies form. On the contrary, with values $A m$ and $M_{S s}$ reduced to a micro-level one can employ the results for calculating the interaction of bodies at micro-level.

\section{SOlution Method}

In Galactica, differential equations of motion (6) are integrated as follows. The value of a function at a next time $T=T_{0}+\Delta T$ is calculated using a Taylor series; this series, for instance, for the coordinate $x$ is

$x=x_{0}+\sum_{k=1}^{K} \frac{1}{k !} x_{0}{ }^{(k)}(\Delta T)^{k}$,

where $x_{0}{ }^{(k)}$ is the derivative of order $k$ at the time $T_{0}$.

The value of velocity $x^{\prime}=v_{x}$ is calculated by a similar formula, and the acceleration $x_{0}{ }^{\prime}=\frac{d v_{x i}}{d T}$, by (6). Higher derivatives $x_{0}{ }^{(k)}$ are calculated using analytical expressions that we had derived by differentiating (6). Presently, a six-order calculation scheme is used in Galactica, i.e. $K=6$. Here, the sixth derivative is calculated from the difference of the fifth derivatives. Addition of each derivative, except for the sixth one, improves the solution accuracy by three orders. The sixth derivative improves the accuracy by 2.5 orders. This method ensures a high accuracy and a good solution dynamics. For instance, in double-length calculations (17 decimal digits) the inaccuracy does not grow in value as calculations are continued.

In adjusting the integration procedure, more than ten various methods for controlling accuracy were tested [7, 8]. Some of those methods are used in Galactica for monitoring the inaccuracies. Data concerning the accuracy are issued in output files. Some relevant data were reported in [7, 8] and in other publications; such data will also be discussed below in other sections. From problem solution data, the user can judge the accuracy and, if necessary, take measures to improve it.

In the present paper, the Galactica algorithm is not described, some elements, however, will be outlined below as they soon as they become necessary for discussion of integration data.

\section{INPUT FILE GALACF26.DAT}

\subsection{General description}

Table 1 lists enumerated parameters involved in the input file galacf26.dat. The numbers of parameters are given in column 1 of the table. All parameters can be subdivided into four groups: 1) interaction region parameters; 2) general problem solution parameters; 3) parameters regulating the output of the trajectories of separate bodies; 4) parameters defining on-screen data display.

The digits with point in the fourth column of Table 1 are real quantities, and the digits without point are integer numbers. The real values are represented in format F11.4; the integer numbers Klmax, Ltk, Ltk2, k2, Kl3, Kl11, and $\mathrm{Kli}$, in format I12; and others, in format I5. The string variable is used in format A12.

In the second column of Table 1, first row, the date of creation of the file version is additionally given, for instance, 01.02.2012.

TABLE I. CONTENTS OFTHE INPUT FILE

\begin{tabular}{|l|l|l|l|}
\hline No & Parameter & Designation & $\begin{array}{l}\text { Digit starting from } \\
\text { the } 60^{\text {th }} \text { position }\end{array}$ \\
\hline 1 & Length of region along axis X $(\mathrm{A}=1) 01.02 .2012$ & $\mathrm{~A}$ & 1. \\
\hline 2 & Height of region along axis $\mathrm{Y}(\mathrm{B} \leq 1)$ & $\mathrm{B}$ & 1. \\
\hline 3 & Thickness of region along axis $\mathrm{Z}(\mathrm{C} \leq 1)$ & $\mathrm{C}$ & 1. \\
\hline 4 & Initial X coordinate of region & $\mathrm{X} 0$ & 0 \\
\hline 5 & Initial Y coordinate of region & $\mathrm{Y} 0$ & 0 \\
\hline 6 & Initial Z coordinate of region & $\mathrm{Z} 0$ & 0 \\
\hline 7 & Number of divisions over axis X & $\mathrm{Mu}$ & 2 \\
\hline 8 & Relative initial density of region & Ro0 & 1. \\
\hline 9 & Relative initial density of point body & RoS & 2. \\
\hline 10 & X coordinate of a separate body & $\mathrm{Xs}$ & 1.5 \\
\hline
\end{tabular}




\begin{tabular}{|c|c|c|c|}
\hline 11 & Y coordinate of a separate body & Ys & 0.5 \\
\hline 12 & Z coordinate of a separate body & $\mathrm{Zs}$ & 0.5 \\
\hline 13 & Velocity of the separate body along axis X & Us & -0.4 \\
\hline 14 & Velocity of the separate body along axis Y & Vs & -0.15 \\
\hline 15 & Velocity of the separate body along axis $\mathrm{Z}$ & Ws & 0.5 \\
\hline 16 & Angular velocity of rotation of region & wu & 0.0 \\
\hline 17 & Relative X coordinate of the axis of rotation & $\mathrm{X} 01$ & 0.5 \\
\hline 18 & Relative Y coordinate of the axis of rotation & Y01 & 0.5 \\
\hline 19 & Absolute average density of region, $\mathrm{kg} / \mathrm{m}^{\wedge} 3$ & Roa & 1.E-7 \\
\hline 20 & Absolute density of bodies, $\mathrm{kg} / \mathrm{m}^{\wedge} 3$ & Rob & 5000. \\
\hline 21 & Depiction with respect to the first body (see below) & $\mathrm{K} 110$ & 0 \\
\hline 22 & Integration time step at $\mathrm{Kl} 1=0$ & $\mathrm{dt}$ & $5.0 \mathrm{e}-4$ \\
\hline 23 & Maximum dimension of TR array & Klmax & 6000 \\
\hline 24 & Number of integration steps in one cycle & Ltk & 1000 \\
\hline 25 & Number of cycles & Ltk2 & 1 \\
\hline 26 & Key: input data from file 4 & K11 & 2 \\
\hline 27 & Key: start of counting time & $\mathrm{Kl} 4$ & 1 \\
\hline 28 & Search of close approaches for body No. at Kl3 & Nbd & 0 \\
\hline 29 & Key: writing data to file & $\mathrm{K} 17$ & 0 \\
\hline 30 & Number of interacting bodies & $\mathrm{k} 2$ & 12 \\
\hline 31 & Interval of data (picture) output (in steps) & $\mathrm{Kl} 3$ & 500 \\
\hline 32 & Beginning of step-by-step data (picture) output & Kl11 & 0 \\
\hline 33 & Key: writing trajectory parameters & Kl12 & 0 \\
\hline 34 & Interval of trajectory parameter output (in steps) & Kli & 50 \\
\hline 35 & Display mode & Nre & 0 \\
\hline 36 & Correction of area size on the screen & $\mathrm{K} 114$ & 1 \\
\hline 37 & Coefficient of area form on the screen & af & 0.72 \\
\hline 38 & Coefficient of mass size of the screen & $\mathrm{Rd}$ & 0.08 \\
\hline 39 & Coefficient of velocity on the screen & amv & 3. \\
\hline 40 & Correction factor of step dtk at merging of bodies & bt & 0.8 \\
\hline 41 & Flat depiction of layer to $\mathrm{Z}$ & $\mathrm{Ke}$ & 0 \\
\hline 42 & Rotation of area relative to $\mathrm{X}$ axis through angle & al & 10. \\
\hline 43 & Rotation of area relative to $\mathrm{Y}$ axis through angle & bet & 20. \\
\hline 44 & Beginning of screen area along $\mathrm{x}$ & $\mathrm{Xe} 0$ & 300. \\
\hline 45 & Beginning of screen area along y & $\mathrm{Ye} 0$ & 280. \\
\hline 46 & Image size in $\mathrm{x}$ & aix & 150. \\
\hline 47 & Image size in $y$ & biy & 70. \\
\hline 48 & Initial data file name & FIMA4 & sn2010Ap.dat \\
\hline 49 & Number of bodies in trajectory analysis & $\mathrm{Np}$ & 2 \\
\hline 50 & Key: body velocity output & $\mathrm{K} 115$ & 0 \\
\hline 51 & Velocity color of the first body & $\operatorname{ipp}(1)$ & 3 \\
\hline 52 & Free key of the first body & jpp(1) & 1 \\
\hline 54 & Number of the first body & $\mathrm{kpp}(1)$ & 1 \\
\hline 55 & Velocity color of the second body & $\operatorname{ipp}(2)$ & 5 \\
\hline 56 & Free key of the second body & $\mathrm{jpp}(2)$ & 1 \\
\hline 57 & Number of the second body & $\mathrm{kpp}(2)$ & 2 \\
\hline 58 & Velocity color of the third body & $\operatorname{ipp}(3)$ & 8 \\
\hline 59 & Free key of the third body & jpp(3) & 1 \\
\hline 60 & Number of the third body & $\mathrm{kpp}(3)$ & 4 \\
\hline
\end{tabular}

\subsection{Interaction region parameters}

The first group, lines $1 \div 20$ and 22 in the table, comprises parameters for setting input data and initial conditions of interacting bodies resulting from a uniform distribution of substance in a certain space region. The region is defined in lines 1-3 of Table 1 as a parallelepiped with sides $\mathrm{A}, \mathrm{B}$, and $\mathrm{C}$. The side lengths are relative values, normalized by the largest length $\mathrm{A}$, so that $\mathrm{A}=1$. The origin has coordinates $\mathrm{X} 0, \mathrm{Y} 0$, and $\mathrm{Z} 0$. Along the axis $\mathrm{X}$, the region is partitioned into $\mathrm{Mu}$ intervals. The lengths $\mathrm{B}$ and $\mathrm{C}$ are also divided into intervals, identical in length and close in value to the partitioning intervals for the side $\mathrm{A}$. The mass of the whole region is defined by the volume A.B.C.

Apart from bodies obtained by partitioning the region into identical volumes, a separate massive body can be specified. Its mass is defined by the relative density RoS (line 9). The value of RoS is normalized by the relative density of the region Ro0, so that Ro0 $=1.0$. The parameters Xs, Ys, and Zs define the relative coordinates of the separate body. If the separate body is located at a distance exceeding the separation between the existing bodies, then this body is added to the total number of bodies.

The radii and masses of bodies are calculated from their volumes and absolute density Rob assuming the bodies spherical. The coordinates of bodies are specified at nodes, the velocities being set zero. The velocity of the separate body is defined by parameters Us, Vs, and Ws. The masses of all bodies are normalized by the mass of the whole system.

In specifying the initial velocities of bodies, a possibility to set rotation of the whole region at an angular velocity wu is provided. The position of the axis 
of rotation is defined by the coordinates $\mathrm{X} 01$ and $\mathrm{Y} 01$. The axis of rotation is parallel to the $\mathrm{Z}$-axis.

For integration of such problems, the integration step is defined in line 22 as the parameter dt. The whole system of initial conditions for bodies is to be generated in the center-of-mass system. However, with an additional separate body introduced, the system of bodies no longer remains attached to the center of mass. As a result, due to the interaction in the system, a directional motion may emerge.

\subsection{General problem solution parameters}

This group of parameters is contained in lines 21 , $23 \div 32,40$, and 48 of Table 1 .

Let us begin with line 24; line 23 will be considered below. The quantity Ltk is the total number of integration steps. This number should be smaller than the maximum value of double-precision integer numbers, 2147483647. If necessary, a greater amount of steps can be set in parameter Ltk2 $>1$. This parameter defines the number of cycles to be performed repeatedly, each cycle comprising Ltk steps.

Key Kl1 takes five values: 0, 1, 2, 3, or 4. With Kl1 = 0 , the initial condition file is not used, and all problem data are defined by parameters described in Sec. 5.2. With $\mathrm{Kll}=1$, input data and initial conditions are read out from the output file garez28.dat. Here, previous problem solution is being continued. However, the accuracy will decrease due to the round-off on passing over from binary to decimal digits at the moment of generation of file garez28.dat. In most cases, such a worsening of accuracy is insignificant. Prior to starting the program, modification can be applied to the step dT in the file garez28.dat. This allows certain calculations to be performed with regulated accuracy.

With $\mathrm{Kl} 1=2$, input data and initial conditions are read out from the file whose name is specified in line 48 as parameter FIMA4. Next two values 3 and 4 of the key Kl1 turn on the calculation continuation mode with unchanged accuracy. Here, after each calculation stage, defined by keys $\mathrm{Kl} 7$ and $\mathrm{K} 13$, an unformatted (binary) file galgon4 is generated. On a new start of the program, reading data out of the input file and initial condition file is ignored; instead, all conditions are read out from the file galgon4. If necessary, modification can be applied to some conditions. For that, an additional code, not a component of Galactica, was written. The values of the keys differ in that during the first run of the program with $\mathrm{Kl} 1$ = 3 solution starts with the file garez28.dat, whereas with $\mathrm{Kl} 1=4$, with the file specified by parameter FIMA4.

In the first run with keys $\mathrm{Kl} 1=2$ or 4 the first time step is reduced by one hundred time, i.e. it is set equal to $0.001 \cdot \mathrm{dT}$.

With $\mathrm{Kl} 4=1$, in line 27 the count of time T starts from the value given in the initial condition file, whereas with $\mathrm{Kl} 4=0$ the time count starts from $\mathrm{T}=0$.
Parameter Nbd specifies the number of the body for which close approaches to minimal distances with other bodies will be recorded in memory over the step interval $\mathrm{K} 13$. In the latter case, a file dice.dat is to be generated, with registering close-approach characteristics after $\mathrm{Kl} 3$ steps; those characteristics include the time, the minimum separation between the bodies, and the number of the approached body. With $\mathrm{Nbd}=0$, close approaches are not registered, no file dice.dat being generated.

With $\mathrm{Kl} 7=1$ in line 29 after $\mathrm{Kl} 3$ steps integration data over intermediate intervals are issued. Those data are accumulated in files $1,2,3 \ldots$ garez28.dat. The total number of generated files is defined as Ltk $\cdot \mathrm{Ltk} 2 / \mathrm{Kl} 3$. When starting a program, one should observe that the number of such files would not appear too large. With $\mathrm{K} 17=0$, no intermediate data outputs are produced.

Parameter $\mathrm{k} 2$ in line 30 defines the number of interacting bodies. If arrays in the executable program has a dimension smaller than $\mathrm{k} 2$, then a message «*08G Change the dimension of arrays $* * *$ Change $12>=\mathrm{k} 2$ » will be output to the display screen and also to the error file ErrGlctca, where $\mathrm{k} 2$ is a set number. In the latter situation, one has to use a program treating a greater number of bodies. It also should be kept in mind that from the initial condition file parameters for $\mathrm{k} 2$ bodies will be read out no matter for how many bodies parameters are available in the file.

In line 31, parameter $\mathrm{Kl} 3$ defines the number of steps following which integration data are output to files: 1, 2, $3 \ldots$, and also to the PC display (or to supercomputer's output stream file). It should be borne in mind that too frequent data output makes the counting rate of Galactica seriously deteriorated.

Key Kl11 in line 32 sets the number of the step on which on during ten steps dT step-by-step data output will be produced. This key makes it possible to thoroughly examine the situation encountered following a long time from the start of calculations. With $\mathrm{Kl} 11=0$, this option is made inactivated.

In line 40, the correction coefficient bt for step dT for merging bodies is specified. The value of bt can be varied if, on an encounter of bodies, the calculation of their merge becomes difficult.

In line 48, parameter FIMA4 defines the name of initial data and condition file. The file name can be specified as a string variable involving maximum of twelve symbols.

\subsection{Parameters for output of trajectories of selected bodies}

For investigation of the trajectory of a selected body one has to know the kinematic characteristics of the body with sufficiently high time resolution. For that purpose, parameters 33-34 and 49-60 are intended. Key Kl12 = 1 in line 33 turns on the option of writing trajectory parameters in a file. Here, kinematic data of certain bodies are to be chosen following a given number of 
steps and after accumulating their amount defined in parameter Klmax, such data are output to trajectory files $1 \mathrm{t}, 2 \mathrm{t}, 3 \mathrm{t} \ldots$ traekt.prn. Parameter Kli defines the number of time steps dT following which kinematic characteristics of bodies are sampled.

Parameter $\mathrm{Np}$ in line 49 defines the total number of the bodies for which trajectory characteristics will be issued. Key Kl15 = 1 in line 50 turns on the option of issuing the velocity components. With $\mathrm{Kl} 15=0$, only the coordinates of the bodies are issued.

In subsequent lines, parameter threes $\operatorname{ipp}(), j p p(), \operatorname{kpp}()$ set a particular body. The number of the body is set by the parameter $\operatorname{kpp}()$. Parameter $\operatorname{ipp}()$ sets the color of the velocity vector in using the Galactica version operating in graphic mode. Parameter jpp() is not used in the present version of Galactica. The digits in round brackets at the above parameters give the number of body sequences in trajectory files $1 \mathrm{t}, 2 \mathrm{t}, 3 \mathrm{t}$... traekt.prn. The total number of such files is defined by parameter Klmax. In the presented compilations of codes the value of Klmax should not exceed 15000. In the same codes the total number of bodies $\mathrm{Np}$ should not exceed three for K115 = 1 , and it should not exceed six at K115 $=0$. For setting additional three bodies, to file galacf26.dat three lines with parameters $\operatorname{ipp}(), \operatorname{jpp}()$ and $\operatorname{kpp}()$ for each body are to be added.

In case of erroneous setting of the above parameters, a massage «*05G Change the dimension of TR *** Kl12d0 $=750, \mathrm{Kl} 12 \mathrm{e} 0=20 »$ is output into the error file ErrGlctca and to the display screen. Here, 750 and 20 are set dimensions of the two-dimensional array for trajectory parameters $\mathrm{TR}(\mathrm{Kl} 12 \mathrm{~d} 0, \mathrm{Kl} 12 \mathrm{e} 0)$, where $\mathrm{Kl} 12 \mathrm{~d} 0$ is the total number of the records, and $\mathrm{Kl} 12 \mathrm{e} 0$ is the number of numerical values registered in one record.

\subsection{On-screen display parameters}

Lines 21, 35-39, and 41-47 in Table 1 specify parameters for display of images of bodies and their velocity vectors (see Figure 1) on the screen. Those parameters are used by programs operating with graphics.

Here, the coordinate system employed in the initial condition file, i.e. the system attached to the center of mass of the whole system, is used. If one wants to represent the motion of a body relative to some other body, then in line 21 the key $\mathrm{Kl} 10$ is to be set to 1 . In the latter case, the coordinate images and the velocity of all bodies will be issued relative to the body whose number is set with parameter $\mathrm{kpp}(1)$ in line 54 .

As it was mentioned above, the range of steps in giving out images is specified with key $\mathrm{Kl} 3$ in line 31. Parameter Nre in line 35 is used to account for the display mode. In the standard graphics FORTRAN the mode of display are given in Appendix 2. The influence of mode depends on the specific computer and executable code. The required value can be selected from submitted ones in Appendix 2 . The value of Nre $=-3$ is probably the most acceptable.
Parameter $\mathrm{Kl} 14$ in a line 36 for maintenance of visibility of bodies at unlimited expansion of system in this version of the program Galactica is not used.

Parameter af in line 37 allows preservation of image shape; i.e., with it, a square is represented as a square, not a rectangle. In representation of a body and its velocity on the screen the sizes on the screen are determined as values normaled to maximum values of mass and velocity. The latter allows one to observe the bodies on variation of their masses and velocities in a broad range during problem solution. For initial representation of a body in variously configured displays correcting coefficients $\mathrm{Rd}$ and amv in lines 38 and 39 are used.

If the body system is represented with a multilayered region over the axis $Z$, then parameter Ke can be used to specify the number of the layers whose bodies will be displayed on the screen. With $\mathrm{Ke}=0$, this option is off.

In lines 42 and 43, rotations of the body system in displaying images relative to axes $\mathrm{X}$ and $\mathrm{Y}$ can be set using respectively parameters al and bet. Both angles are to be expressed in degrees.

In lines 44 and 45, parameters $\mathrm{Xe} 0$ and $\mathrm{Ye} 0$ specify the top left origin of the region to be displayed on the screen. The image scales over the axes $\mathrm{X}$ and $\mathrm{Y}$ can be altered using parameters aix and biy, respectively. To enlarge the image, the parameters aix and biy are to be increased in value.

As it was noted above, the velocity vectors of individual bodies can be highlighted with certain color. The number of the body to be highlighted is set by parameters $\mathrm{kpp}()$ in lines 54, 57, etc., and the number of the color, by parameters $\operatorname{ipp}()$ in lines 51,55 , etc. In standard FORTRAN graphic operators, the correspondence between the color and its number is defined in file FrtnColr.bmp contained in folder InpFiles.

View the image depends on the parameter $\mathrm{Mu}$. It is therefore desirable to set the value of this parameter, as well as the parameters $\mathrm{B}$ and $\mathrm{C}$ in lines 2 and 3 they are in the file of initial conditions. The positions of these parameters are described in section 5.

The form of representation of integration data depends on many factors, including the FORTRAN and compiler version, and also the screen type and screen resolution. Given in folder InCndFls together with reference initial condition files for solved problems are images of bodies contained in bmp-files. The names of those files coincide with the initial condition files. For instance, for the initial condition file sn49jplc.dat the images are contained in the file named Sn49jplc.bmp.

Body images are also available in the folder InpFiles\Area. One can find here six examples of the input file galacf26.dat; in the file names, the extension dat is replaced with other symbols. Those symbols denote the type of problems in which the interaction between bodies formed by a region filled with substance is considered. In such problems, body images are contained in bmp-files, 
where the body names are represented with symbols forming the input file name. For instance, the input file galacf26.64r represents interaction of 64 bodies moving in radial direction, the image being given in file 64r.bmp.

The input files contained in this folder give various examples of using parameters for data display; the outcomes of a particular choice of parameters are easy to trace considering graphic files.

\section{INITIAL CONDITION AND INTEGRATION DATA FILES}

Initial condition files, for instance, files sn49f.dat, sn49jplc.dat, etc. in folder InCndFls, and also output files: 1, 2, 3, etc., and file garez28.dat are identical in structure. In describing the files, we will use designations of two kinds: 1) unsubscribed Roman type, and 2) italic type. The latter designations are used in programs, and the former designations, in printed materials.

Each of the above-mentioned files begins with a set of 24 numbers: T0, omm, Um, dTp, Px, Py, Pz, AMx, AMy, AMz, Spsx, Spsy, Spsz, E, Em, Ett, dT, i2b, j2b, k2b, A, $\mathrm{B}, \mathrm{C}$, and $\mathrm{Mu}$. Those numbers are common to all interacting bodies. Then, fifteen numbers for each $\mathrm{k}$-th body follow: om(k), (X(k,q),q=1,3), (U(k,q),q=1,3), $(\mathrm{dUp}(\mathrm{k}, \mathrm{q}), \mathrm{q}=1,3),(\operatorname{Sp}(\mathrm{k}, \mathrm{q}), \mathrm{q}=1,3), \operatorname{Ra}(\mathrm{k}), \operatorname{Et}(\mathrm{k})$. Here, $\mathrm{q}$ is the subscript listing the variables over the three coordinates $\mathrm{x}, \mathrm{y}$, and $\mathrm{z}$.

The general parameters are the following:

T0 - time in relative units, for instance, in solar-system files in sidereal centuries, each century comprising 36525.636042 days;

omm - maximal mass of body reduced by the total mass of all bodies;

$\mathrm{Um}$ - maximal velocity in relative units;

$\mathrm{dTp}$ - time step in relative units at the previous step;

$\mathrm{Px}, \mathrm{Py}, \mathrm{Pz}-$ projections of the total momentum of the whole body system on the coordinate axes;

$\mathrm{AMx}, \mathrm{AMy}, \mathrm{AMz}$ - projections of the total angular momentum of the whole body system;

Spsx, Spsy, Spsz - projections of the sum of the angular momentum due to self-rotation of bodies around their axes (spin projections);

E - total kinetic energy of all bodies at the given moment T0;

Em - maximal kinetic energy of all bodies starting from the beginning of integration;

Ett - thermal energy of all bodies which the bodies have acquired during their merging on collision;

$\mathrm{dT}$ - current time step in relative units;

$\mathrm{i} 2 \mathrm{~b}, \mathrm{j} 2 \mathrm{~b}, \mathrm{k} 2 \mathrm{~b}$ - number of nodes obtained on partitioning of the substance array over the axes $\mathrm{x}, \mathrm{y}$, and $\mathrm{Z}$, respectively;

A, B, C - dimensions of the array over the axes $\mathrm{x}, \mathrm{y}$, and $\mathrm{z}$;

$\mathrm{Mu}$ - total number of divisions of the array over the axis $\mathrm{x}$.
The last seven parameters are used in setting interacting bodies in the form of a substance uniformly distributed in the space with density Ro0.

Next, 15 parameters for each body follow:

om $(\mathrm{k}) \div m_{o \mathrm{k}}-$ non-dimensional (relative) mass of the $\mathrm{k}$ $=i$-th body ( $\mathrm{k}$ and $i$ are the numbers of bodies in different texts);

$(\mathrm{X}(\mathrm{k}, \mathrm{q}), \mathrm{q}=1,3) \div x_{i}, y_{i}, z_{i}-$ non-dimensional barycentric coordinates of the $\mathrm{k}=i$-th body; for solar-system files in equatorial coordinate system;

$(\mathrm{U}(\mathrm{k}, \mathrm{q}), \mathrm{q}=1,3) \div v_{x i}, \quad v_{y i}, \quad v_{z i}-$ non-dimensional velocities of the $\mathrm{k}=i$-th body;

$(\mathrm{dUp}(\mathrm{k}, \mathrm{q}), \mathrm{q}=1,3) \div x_{i}^{(5)}, y_{i}^{(5)}, z_{i}^{(5)}-$ non-dimensional derivatives of the fifth order for the $\mathrm{k}=i$-th body;

$(\mathrm{Sp}(\mathrm{k}, \mathrm{q}), \mathrm{q}=1,3) \div S_{p x i}, S_{p y i}, S_{p z i}-$ non-dimensional angular momentum (spins) due to the self-rotation of the $\mathrm{k}=i$-th body. A body is assumed to get a spin if the body has formed as a result of the merge of other bodies having approached each other to a distance smaller than the sum of their radii. Initially, the rotation of bodies can be ignored, with their spins being defined as $S_{p x i}=S_{p y i}=S_{p z i}=0$ This strategy is adopted in the initial condition files given below.

$\mathrm{Ra}(\mathrm{k})$ - non-dimensional equivalent radius of the $\mathrm{k}=i$ th body; this radius is calculated from the mass and average density of the body, the body assumed spherical;

$\mathrm{Et}(\mathrm{k})-E_{t i}-$ non-dimensional thermal energy of the $\mathrm{k}=$ $i$-th body. This energy has arisen from merging of several bodies in one body due to transformation of kinetic energy into thermal energy.

Initially, the thermal energy of bodies can be neglected, i.e. it can be set to zero, $E_{t i}=0$, this being the case in the provided initial condition files. As two massive bodies $m_{o i}$ and $m_{o k}$ merge together, the thermal energy of the resultant body is calculated as

$E_{t i}=\frac{m_{o k} m_{o i} v^{2} r k i}{2\left(m_{o k}+m_{o i}\right)}$,

where $m_{o i} \geq m_{o k}$, and $v_{r k i}$ is the non-dimensional radial velocity of the massive body $m_{o k}$ relative to the body $m_{o i}$.

On merging, the mass of the $i$-th body increases, assuming the value $m_{o i}+m_{o k}$, the mass of the $k$-th body being put equal to zero. The changes of coordinates, velocity and spin projections, and body radius due to the merge of bodies are calculated using the algorithm described in Sec. 11.3 of $[4,5])$.

In the last line of the initial condition file, five numbers can be written: date of generation of initial conditions, total number of bodies, mass of the system, scale size in meters, and velocity coefficient: date, k2, $M_{S s}, A m, k_{v}$. The date is represented as an integer number involving eight digits: year, month, day. This line is not read by Galactica, and it is absent from the calculated data file.

In Appendix 1 there is the sample of program in MathCad software for generation of initial condition file. 


\section{TRAJECTORY FiLES 1T, 2T... TRAEKT.PRN}

As mentioned in Sec. 5.4, the names of trajectory files look as 1t, 2t, 3t ... traekt.prn. All the names look similarly except for the last file, traekt.prn. Each record refers to one time; it contains Kl12e numbers. The total amount of the numbers is defined by the expression

$\mathrm{Kl} 12 \mathrm{e}=3 \cdot(\mathrm{Kl} 15+1) \cdot \mathrm{Np}+2$,

where $\mathrm{Kl} 15$ and $\mathrm{Np}$ are parameters specified in the input file galacf26.dat and described in Sec. 5.4.

The record starts from moment of time $\mathrm{T}$ in nondimensional units. Then, the kinetic energy of the whole system $\mathrm{E}$ follows. Then, for each body, three coordinates, $\mathrm{x}, \mathrm{y}$, and $\mathrm{z}$ are issued. With $\mathrm{Kl} 15=1$, also issued are the velocities vx, vy, vz. All those parameters, including time, are represented as non-dimensional quantities (see Sec. 3).

The total number of the records in the files and their volume are defined by the parameters described in Sec. 5.4. If the total amount of records is small, then just one trajectory file traekt.prn will be generated. To the end of the file, a record comprising ten general problem parameters is appended: T, Tem, Em, Ett, AMz1, Nb, Lsls, Um, omm, and Amz,

Here, $\mathrm{T}$ is the time at which the calculation of the problem was over;

Tem is the time at which the kinetic energy of the system was maximal;

Em is the maximum value of the kinetic energy of the system;

Ett is the thermal energy of the system at the end of the calculation;

AMz1 is the angular momentum of the whole system relative to the axis $\mathrm{z}$ at the first calculation step;

$\mathrm{Nb}$ is the total number of bodies in the system at the beginning of the calculation;

Lsls is the number of merges, i.e. the number of bodies that, on approaching one another, formed a single body;

$\mathrm{Um}$ is the highest velocity in the calculation run;

omm is the mass of the largest body;

$\mathrm{AMz}$ is the angular momentum of the whole system relative to the axis $\mathrm{Z}$ at the end of calculation.

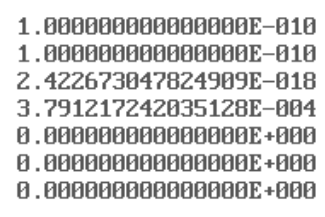

0.

$$
\begin{array}{cr}
9.986599585707880 \mathrm{E}-001 & 15.262213869302140 \\
1.734723475976807 \mathrm{E}-018 & -1.084202172485504 \mathrm{E}-018 \\
1.081760992678619 \mathrm{E}-005 & -1.606331142603044 \mathrm{E}-004 \\
0.000000000000000 \mathrm{E}+000 & 0.000000000000000 \mathrm{E}+000 \\
8.608432591162210 \mathrm{E}-003 & 8.608432591162210 \mathrm{E}-003 \\
1 & 1.000000000000000 \mathrm{E}-007
\end{array}
$$$$
T, m_{\max }, v_{\max }, \Delta t_{p}, P_{x}, P_{y}, P_{z}, M_{x}, M_{y}, M_{z}, S_{x}, S_{y}, S_{z},
$$

If preceding files, e.g. a file $1 \mathrm{t}$, are output, then the file raekt.prn has the same volume, and after the final record from the preceding file.

In Galactica, the kinetic energy of the system is alculated as

$E=\sum_{i=1}^{n} 0.5 m_{i} v_{i}^{2}$

As it was noted above, on merging of two bodies the is calculated by (11). As it will be shown calculated by (14).

\section{Close-ApProAch FILE DiCE.DAT}

As it was mentioned in Sec. 5.3, if the parameter Nbd to be written in the file dice.dat: time at which a one another. The total number of such records in the file istk.Ltk2/Kl3.

We used this option while investigating into the and Figure 7 in [10] show data obtained on the basis of

In solving a problem on a PC with a Galactica program with graphic option, numerical data concerning the following a certain number of integration output to the screen. In solving the problem on a supercomputer, the data are issued to the output stream file.

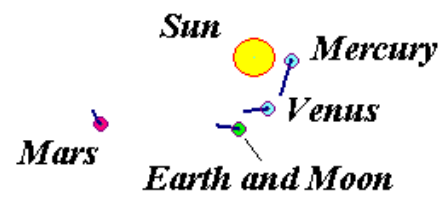

Figure 1. On-screen representation of integration data for equation system (6) as viewed from the South Pole. Explanation and denotation of numerical parameters are given in italics. 
The first 16 parameters reproduce the values of common parameters:

TO, omm, Um, dTp, Px, Py, Pz, AMx, AMy, AMz, Spsx, Spsy, Spsz, E, Em, Ett, used in the initial condition and integrated data files described in Sec. 5. The remaining three parameters are the integration step number $L_{t}$, the value of this step $d T$, and the change of the angular momentum $\delta M_{z}$ taken in projection onto the axis $z$ (it should be remembered here that the total number of the integration step involves the product $L t k \cdot L t k 2$ ).

Those quantities enable an analysis of the evolution of the system and the integration process of (6). Each of the quantities, the largest mass omm $=m_{\max }$, the momentum $P$, the angular momentum $M$, the sum of intrinsic angular momentum $S$, the kinetic energy $E$, the thermal energy $E_{t}$ of all bodies, and the relative change $\left(\delta M_{z}\right)$ of the projection of the angular momentum $M$ onto the axis $z$, allow the user to get an idea how the solution evolves and, in case of an error, to spot the error.

An important control factor is the relative change of $\delta M_{z}$. In the absence of external actions, we have:

$M_{z}=\sum_{i=1}^{n} m_{i}\left(v_{y i} x_{i}-v_{x i} y_{i}\right)=$ const .

That is why the relative change of the angular momentum

$\delta M_{z}=\left(M_{z}-M_{z 0}\right) / M_{z 0}$,

should be zero (here, $M_{z 0}$ is the value of the angular momentum at the initial time). If the angular momentum appears to be non-zero, the non-zero value should be considered as a consequence of some inaccuracy having occurred in numerical integration of the problem. More details about the inaccuracy of $\delta M_{z}$ and the relation of this inaccuracy with the inaccuracies of coordinates and velocities can be found in $[7,8]$.

Non-zero values of the momentum $\mathrm{Px}, \mathrm{Py}$, or $\mathrm{Pz}$ for the whole system may point to an occurrence of an inaccuracy in some particular direction. Extraordinarily high values of the maximum mass omm and velocity Um will suggest the user a likely source of inaccuracies.

\section{EXAMPles OF InPUT Files GalaCF26.DAT}

The folder InpFiles contains examples of input files: such files can also be found in the subfolder Area. The input file galacf26.dat designed to solution of the problem of 3000 axisymmetric bodys interaction with a file of initial conditions axsymb3t.dat. An input file galacf26.v01 was used in solving the problem on multilayered ring structures [3]. The file FrtnColr.bmp gives the color numbers, from 1 to 12 , adopted in standard FORTRAN graphics. Those colors can be set for displaying the velocity vectors of certain particles. As it was mentioned previously, the colors are set with parameters $\operatorname{ipp}()$ in the input file.

The folder Area gives six examples of input file galacf26.dat with setting initial conditions for interacting bodies obtained by partitioning a substance region into a certain number of bodies. Instead of the extension «dat», the names of the files contain symbols identifying the body system obtained. For each of the input files, a graphic bmp-file is also provided, whose name begins with the same symbols. Below we give a list of the problems represented with those files:

1) galacf $26.3 \mathrm{br}$ - radial interaction involving three bodies;

2) galacf $26.3 \mathrm{~b} 1$ - three bodies with specified angular rate of rotation of the initial region;

3) galacf26.4bo - four bodies with specified rotational velocity;

4) galacf $26.64 \mathrm{r}$ - radial interaction involving 64 bodies;

5) galacf $26.64 \mathrm{w}$ - interaction of 64 bodies at specified rotational velocity of region;

6) galacf $26.65 \mathrm{~b}$ - interaction of 65 bodies, of which 64 bodies are represented with substance distributions in a region and the remaining body resides at a distance from the region.

Samples of input files galacf26.dat are available also in a folder InCndFls Preprtn for start on the account of three samples of files of the initial conditions: axsymb30.dat, axsymb3c.dat and axsymb3t.dat in a graphic mode of the account.

\section{Initial Condition Files for SeVEral Solved PROBLEMS}

The folder InCndFls contains initial data and initial condition files for some solved problems. The data structure in those files was described in Sec. 6. Here, additional information about the use of such files is given.

The folder contains files of three types: 1) initial condition files; 2) body image files, named similarly to the initial condition files; 3) input files galacf26.dat, whose names are formed by symbols g26 and by the names of the initial condition files. Files of the last type are presented only for several typical problems.

Files sn49f.dat and sn49jplc.dat were used to investigate into the evolution of the Solar system over a time interval of 100 million years [7, 8]. The files contain the masses, the coordinates, and the velocities of the planets, the Moon, and the Sun by the date 30.12.1949 with Julian day JD $=2433280.5$. The data in the file sn49jplc.dat were borrowed from later Jet Propulsion Laboratory (JPL) ephemeredes in comparison with sn49f.dat, namely, from DE406 ephemeredes. That is why data in sn49jplc.dat more accurately reflect the properties of the Solar system. The files contain data for eleven bodies. In addition, a line for the twelfth body is reserved. To this line, data can be input for a flying vehicle or an asteroid whose motion in the Solar system is to be investigated.

The file sn69JPA2.dat contains initial conditions by the date June 28, 1969 with JD = 2440400.5. Apart from the mentioned bodies, this file additionally contains data for three asteroids: Ceres, Pallas, and Vesta, so that we have 
a total of fourteen bodies. The masses, the coordinates, and the velocities in that file were borrowed from the reference data of DE405 ephemerides [11]. In comparison with sn49f.dat and sn49jplc.dat, calculation by that file yields a better agreement of calculated data with the DE406 ephemerides for the planets from Jupiter to Pluto.

The files sn49e5n.dat, sn49e5n2.dat and sn49e5n3.dat contain data for three compound models (1-st, 2-nd, and 3-rd) of Earth rotation [7, 12]. The data for the eleven solar-system bodies precede the data for the bodies employed in the Earth rotation model. Their parameters are based on the same data as the parameters in sn49f.dat are, except for the Earth mass. In those files, the mass of the central body of the compound model of the Earth is reduced by the mass of the peripheral bodies considered in the model.

The files sn49S6J4.dat and sn49S6J5.dat contain data for the 4-th and 5-th compound models of the Sun [1316]. Appearing first are data for the eleven solar-system bodies. Those data are based on the same sources as the parameters in sn49jplc.dat, except for the Sun mass. The mass of the central body in the Sun model is reduced by the mass of peripheral bodies involved in the model. Then, parameters of the peripheral bodies appear. In sn49S6J5.dat, the model involves ten bodies, and in sn49S6J4.dat, it involves five bodies.

The file sn49S6J6.dat contains initial data and initial conditions for the problem about the action of the compound Sun model on Mercury [17]. The file involves two objects, Mercury and the compound model of the Sun, the latter model involving a central body and ten peripheral bodies.

The files sn08AsAp.dat and sn08AsDA.dat [9, 10, 1821] contain data about the eleven solar-system bodies and, in addition, asteroids Apophis and 1950 DA by the date 30.11 .08 with Julian day JD $=2454800.5$. The masses of the eleven solar-system bodies here are the same as in sn49jplc.dat. The coordinates and velocities of the bodies are based on the DE406 ephemerides. The coordinates and the velocities of the asteroids were calculated by an original procedure of [10] from the orbital elements of the asteroids borrowed from the NASA database of small bodies [22, 23].

In Sn2010Ap.dat, the data for the Solar system and Apophis are taken by the date 04.01.2010 with Julian day $\mathrm{JD}=2445200.5$. The masses are the same as in sn49jplc.dat. The coordinates and the velocity of Apophis were borrowed from the same sources as in previous files (see [10]).

The files snKo2102.dat and snKo2110.dat contain initial data and conditions for models of three-layered ring structures, the second and tenth ones, respectively [3, 24]. The mass of the body system is taken equal to the mass of the entire Solar system. The former file predicts a stable structure with the mass of the central body being equal to the Sun mass, and the latter file predict an unstable structure with the mass of the central body equal to half the Sun mass.

As noted above, all the parameters in the files are given in non-dimensional form: the masses are normalized by the mass Mss of the body system in kilograms; the sizes, by the characteristic size Am of the system in meters; and the velocity is multiplied by the coefficient $\mathrm{kv}$ expressed in $\mathrm{sec} / \mathrm{m}$. For solar-system problems the non-dimensional time is expressed in sidereal centuries, and the barycentric equatorial coordinate system by the epoch 2000.0 is adopted. For sn49f.dat, the epoch 1950.0 is adopted. To the end of the file, an information line is appended indicating the date of creation of initial conditions, the number of involved bodies, and also the values of Mss, Am, and kv.

Apart from the above-listed files contained in folder InCndFls, other three initial condition files axsymb30.dat, axsymb3c.dat, and axsymb3t.dat are available; those files refer to axisymmetric interaction of 30, 300, and 3000 bodies, respectively. Those files, contained in folder Preprtn, can be used for testing Galactica codes. Besides in this folder there are the samples of input files galacf26.dat for start on calculation of the mentioned three files of the initial conditions. The input file settings are for a good presentation of the results in graphical mode account. The parameters for good representation of results in graphic mode of the calculation are established in the input files.

\section{EXECUTABLE FILES}

The folder RunFiles contains executable files. Table 2 lists the names of the main exe-files for PC and for a Unix OS supercomputer. In the names, the following agreements are adopted. Symbols glc mean Galactica. Digit 3 means a third version of the program intended for common use; the last number of the version in individual use was $34 \mathrm{a}$. The character «b» with subsequent symbols means the total number of bodies.

Executable files without graphics compiled by FORTRAN PowerStation v4.0. Codes with graphics, which are compiled by the Compaq Visual FORTRAN Professional Edition v6.5.0 in FORTRAN standard graphics, have in a name the letter $\mathrm{p}$.

In addition to the files listed in the Table 2, the folder RunFiles contains exe-files with graphics glc 3 cb30.exe and glc3b1c2.exe capable of calculating respectively 30 and 120 bodies. The codes were compiled using Microsoft FORTRAN Compiler V.5.00; they can be run on a PC under the outdated DOS.

\section{TABLE II. NAMES OF MAIN EXE-FILES OF GALACTICA}

\begin{tabular}{|l|l|l|l|}
\hline \multirow{2}{*}{$\begin{array}{l}\text { Number } \\
\text { of } \\
\text { interacting } \\
\text { bodies }\end{array}$} & $\begin{array}{l}\text { Without } \\
\text { graphics }\end{array}$ & $\begin{array}{l}\text { With } \\
\text { graphics }\end{array}$ & $\begin{array}{l}\text { For a UNIX OS } \\
\text { super-computer }\end{array}$ \\
\cline { 2 - 4 } & glc3b30.exe & glc3pb30.exe & glc3b30; glc3b30e \\
\hline 30 & glc3b3c.exe & glc3pb3c.exe & glc3b3c \\
\hline 300 & glc3b3t.exe & glc3pb3t.exe & glc3b3t \\
\hline
\end{tabular}


The code named glc3b30e capable of calculating 30 bodies on a supercomputer operates with extended digit length (34 decimal symbols). This program can read initial condition files with double length numbers, and it generates integration data files with digits of quadruple length. The speed of calculation with this program is 33 times lower in comparison with other codes. It makes sense to use this version of Galactica in solving such problems as encounter of an asteroid with a celestial body or for calculating the motion of spacecrafts involving launch and landing trajectory portions, i.e. than a close approach of bodies is involved in the problem. All codes except for the latter one use double length numbers.

\section{GENERATION OF INITIAL CONDITION FILE}

After getting acquainted with the preceding sections, the user is ready to tackle its own problem. In solving each problem, tasks may be encountered whose solution will require accomplishment of some other subtasks. A few such problems were solved in [3-5, 7, 12-21, 24-25]. All problems having been resolved and the configuration of the body system and the initial motion, defined, the user can proceed with generation of an input data and initial condition file. As applied to the problem of axisymmetric interaction of $N$ bodies [2], the file InCnPrpr.mcd exemplifies a procedure for generation of an initial condition file. The sample is written in MathCad software (see Appendix 1). This sample can be implemented using any programming language or mathematical software. Below, taking the sample as an example, we will comment on the particular actions. In the problem of interest, the following configuration of interacting bodies is defined: around a central body, peripheral bodies are located. The velocities of the bodies are chosen such that the bodies move along elliptic orbits with eccentricity e.

Item 13.1 of Appendix 1 defines the total number of interacting bodies $\mathrm{Nb}$ and the lengths of indexes $\mathrm{i}$ and $\mathrm{i} 1$. Since an index begins with zero, the largest value of $\mathrm{N}$ is smaller than $\mathrm{Nb}$ by unity.

Item 13.2 defines the main constants, namely, the constant of gravitation $\mathrm{G}$, the astronomical unit AU, the sidereal year in days $\mathrm{P}_{\mathrm{sd}}$ and in seconds $\mathrm{P}_{\text {sdsc }}$.

Item 13.3 defines the Earth mass $\mathrm{mEr}$, the Sun mass $\mathrm{M}_{\mathrm{S}}$, the mass of the Solar system $\mathrm{M}_{\mathrm{Ss}}$, the body densities Roba, the major semi-axis of the orbit of the peripheral bodies (a), the eccentricity of the orbit (e), and the inclination $\varepsilon$ of the orbit plane to the xy-plane, which is assumed to be the Earth's equatorial plane.

In 13.4, for all the interacting bodies, masses in kilograms and radii in meters are calculated.

Then, in item 13.5 the algorithm of $[2,4,5]$ is implemented to determine the coordinates and velocities of the peripheral bodies in the orbit plane. Here, the notation introduced in our book «The Theory of Interaction» [4-5] is used: $\alpha_{1}$ is the trajectory parameter, $\mu_{1}$ is the interaction parameter, and the pericentre parameters are $R_{p}$ and $v_{p}: R_{p}$ being the radius and $v_{p}$, velocity.

This algorithm is intended for calculating the interaction of an arbitrary number of bodies. That is why one has to check that the orbit will not be overcrowded with the bodies. This check can be performed as follows: the separation between two bodies do reduced by the sum of their radii should be less than 50. Otherwise in Sec. 13.1 the major semi-axis value "a" should be increased using the coefficient $k_{a}$.

The problem of axisymmetric interaction of bodies was solved exactly in $[2,4,5]$. Here, the peripheral bodies move along ellipses. In item 13.6 those orbits are calculated, and they are plotted in coordinates ynb-xnb. The index $\mathrm{i} 2=1,3 . \mathrm{N}$ in MathCad can be used to call for plotting graphs not for all bodies. Here, orbits are to be plotted for each third body. Those graphs confirm that the set configuration of interacting bodies is indeed axisymmetric, and the bodies move in an elliptic orbit with the required eccentricity e.

In item 13.7, the orbit plane rotates through an angle $\varepsilon$ relative to the axis $\mathrm{x}$. Here, differential equations (6) «work» along all the three axes, $\mathrm{x}, \mathrm{y}$, and $\mathrm{z}$, despite the fact that the initial statement of the problem was planar. Due to the latter rotation, to 20 trustworthiness criterions considered in [7, 8], one more criterion can be added. If integration of (6) yields orbits deflected from the initial plane, then the deviations should be identified as inaccuracies of the integration method.

In item 13.8, we pass to non-dimensional variables. Here, $\mathrm{ms}_{\mathrm{N}+1}$ is the sum of the masses of all the bodies involved in the system, Am is the scaling length factor in meters, and $\mathrm{k}_{\mathrm{t}}$ and $\mathrm{k}_{\mathrm{v}}$ are the scaling time and velocity factors expressed in SI units. Those factors were described in Sec. 3.

In the latter example, the scaling factor Am is calculated from the condition that the non-dimensional time unit should be equal to sidereal century $100 \cdot \mathrm{P}_{\mathrm{sd}}$. Since Am is determined with some non-zero inaccuracy due to extraction of a cubic root, then a procedure for refining the quantity Am is to be applied.

In item 13.9, according to formulas (4)-(5) the variables are reduced to the center of mass of the system. Here, for instance, $\mathrm{Xc}_{\mathrm{N}+1}$ and $\mathrm{Vxc}_{\mathrm{N}+1}$ are the coordinate and the velocity of the center of mass. In the problem of interest, initially the center of mass is at the origin, so that the above values are both close to zero. Nonetheless, the procedure should be accomplished, and the parameter values of the center of mass, examined. The latter examination is a check of the algorithm for calculating initial conditions. In the present case, the values of the parameters are within $4 \cdot 10^{-19}$. That is why for performing Galactica calculations with a lower error preparation of initial conditions with a better accuracy is required. For instance, it would be desirable that the initial conditions be prepared with a larger number length than it can be made in MathCad. 
In item 13.10, projections of the angular momentum $\mathrm{Mx}_{\mathrm{N}+1}, \mathrm{My}_{\mathrm{N}+1}$, and $\mathrm{Mz}_{\mathrm{N}+1}$ of the whole body system and the absolute value of the angular momentum M0 are calculated. For the problem of interest, the total angular momentum of the whole system can also be calculated as

$M 00=(N b-1) \cdot m_{1} \cdot R p \cdot v p$.

Then, the check is to be performed. The sum of nondimensional masses of the system should equal unity. We determine the relative error of the angular momentum M0 and its value M00 calculated by formula (16), and also the relative inaccuracy of the scaling factors $\mathrm{Am}, \mathrm{k}_{\mathrm{t}}$, and $\mathrm{k}_{\mathrm{v}}$. Those checks enable evaluation of the inaccuracy of the algorithm, and also the value of the computational inaccuracy. As it is seen, here the residual of the scaling factors is zero. This result was obtained through refinement of Am in item 13.8.

In item 13.11, we calculate the number $\mathrm{Mu}$ to be used in the input file galacf26.dat for specifying initial conditions for space-distributed substance. Here, the parameter $\mathrm{Mu}$ is not used. However, for right representation of bodies in graphic mode this number should be made consistent with the number of bodies $\mathrm{Nb}$. The table below exemplifies matched parameters for different values of $\mathrm{Nb}$ :

\begin{tabular}{|l|l|l|l|}
\hline $\mathrm{B}$ & $\mathrm{C}$ & $\mathrm{Mu}$ & $\mathrm{Nb}$ \\
\hline 0.5 & 0.25 & 4 & 30 \\
\hline 0.9 & 0.6 & 7 & 300 \\
\hline 0.9 & 0.7955 & 15 & 3000 \\
\hline
\end{tabular}

In item 13.12, initial data and conditions are written in a prn-file, for instance, axsymb30b.prn. The file contains 15 columns. The first two lines contain general data involving 24 parameters. That is why six zeroes at the end of the second line are to be removed, and the file is to be renamed in a dat-file, for instance, axsymb30b.dat.

After the first two lines, additional lines, each comprising 15 values, appear. The total number of the lines is equal to the total number of the bodies involved in the problem. At the end, there appears an information line, whose first number is the date of creation of the initial condition file: year.mm.dd.

The next two graphs show the coordinates and velocities in the body system. Those values enable a check of the sought configuration planned in the problem statement. As it is seen from the graphs, the coordinates and velocities of the bodies form an axisymmetric pattern; in other words, they correspond to the problem of axisymmetric interaction of the bodies.

The above-considered program InCnPrpr.med was used to generate the following files: axsymb30.dat, axsymb3c.dat, and axsymb3t.dat for 30, 300, and 3000 bodies, respectively. Those files permit check of operability of the exe-files, evaluation of the total errors in solving the problem, and integration step adjustment ensuring a required accuracy of problem solution.

At the end of the sample program, two more points are outlined which may appear necessary is setting initial conditions. In item 13.13, an algorithm for calculating the number of steps Ltk and the corrected time step value $\mathrm{dTc}$ for exact solution of the problem from one date with Julian day JD1 to another date with Julian day JD2 are considered. The need in the calculation arises in solving problems on interaction of solar-system bodies.

If the non-dimensional time in Galactica calculations is expressed in sidereal centuries, then the time difference $\Delta \mathrm{Tjd}$ in Julian days is transformed into the number $\Delta \mathrm{T}$ in sidereal days. Given the step dT, we calculate the number of steps Ltkr to be rounded to a close value Ltk. Then, the corrected step dTc is to be determined.

Here, the calculations were performed for two variants: (i) in resumed calculation mode (with key $\mathrm{Kl} 3$ set to 1 or 3 ), and (ii) in initial calculation mode (with key $\mathrm{Kl} 3$ set to 2 or 4 ). In the latter case, as it was mentioned previously, the calculation at the first step is to be performed with the step $0.001 \cdot d T$.

At the end, check of the calculated values of Ltk and $\mathrm{dTc}$ is to be performed.

Item 13.14 gives an algorithm for reading out an initial condition file or an integration data file of type garez28.dat. This becomes needed in setting initial conditions from a calculated data file of an analogous problem. Besides, that algorithm can be used for analyzing the output data.

In the first line, the number of bodies $\mathrm{Nbg}$ and index lengths ig and ig2is are defined. In the first line, the number of bodies is set. Here, instead of the operator READPRN, the operator READ is used. The latter operator permits reading-out of a non-ordered file. After the reading, required quantities can be displayed as shown in the graph.

\section{XIV.Problem Solving Procedure}

After the user has made his decision concerning the configuration of the system of interacting bodies, their masses, sizes, and initial conditions, including the coordinates and velocities of the bodies, he has to proceed with generation of an initial condition file. For passage to non-dimensional variables, one has to choose, in addition to the mass of the system $\mathrm{M}_{\mathrm{Ss}}$, one more scaling factor, namely, the distance Am or a nondimensional time unit. In the latter case, the quantity Am will be defined using the non-dimensional time.

Next, in accordance with Sec. 13, one has to produce an initial condition file, for instance, a file named initial.dat. In generating the latter file, one has to introduce into the algorithm, where possible, all checks (in Sec. 13, several such checks were outlined), so that to achieve full compliance of file parameters with the determined configuration of the problem. As it was shown in Sec. 13, it would be highly desirable to output the configuration of body positions and velocities in graphic form. With a large number of involved bodies, the latter offers a most reliable checking strategy. Then, one has to generate numerical values for the input file 
galacf26.dat. In Sec. 5, a description of the parameters entering that file is given and their functional meaning is indicated. For first starts of the problem, only necessary functions are to be used. Subsequently, as may be necessary, other functions, one by one, can be added.

Then, the user is invited to choose an exe-file from folder RunFiles. For instance, if, in the system of interest, the total number of interacting bodies is $\mathrm{k} 2=29$, then the exe-file intended for 30 bodies is to be used. An exe-file intended for treating a greater number of bodies can also be employed. Yet, such an exe-file will use more RAM memory space; in some cases this circumstance will slow down the solution of the problem.

If the user solves a problem with the help of Galactica for the first time, a most appropriate strategy would be to choose from the folders InCndFls, Preprtn, and InpFiles a sample input file and a sample initial condition file, and then test the operation of the chosen exe-file in various modes of operation.

Then, the user may start its own problem: first over one step and, then, over a few steps. Here, errors in readingout of the input file or the initial data file can be encountered. In the latter case, the user must use another program, e.g. the program InCnPrpr.mcd, to read out the files with the operator READ, and get output the values of all parameters. As a rule, errors in reading out the input file can be identified through comparison with a similar readable file.

If the running time of the program is long, the user has to perform calculations over short running time to determine the time required for executing one step and, then, he has to evaluate the time required for solving the whole problem. Then, the user has to calculate, using the formulas given in Secs. 5 and 6, the total volume of output files: 1, 2, $3 \ldots$ garez28.dat, and 1t, 2t, $3 \mathrm{t} \ldots$ traekt.prn. It is also necessary to check that this volume does not exceed the volume allocated for the solution of the problem.

After the start of the exe-file, through observation of data on the display screen or data issued in the output stream file (see Figure 1) the user can monitor the solution process. For the user, each of the 20 issued parameter described in Sec. 9 can serve an indicator of the specific features of the problem. The behavior of those parameters can be helpful in revealing an error in the problem statement or, on the contrary, this behavior can justify the problem statement, or even help in predicting the final result.

For analysis, data should be represented in graphic form. To be analyzed is the variation of the coordinates of individual bodies in time, i.e. the trajectories of the bodies. If the trajectories are cyclic, then the orbit elements of bodies should be identified, and the evolution of elements in time, analyzed.

In our previous publications, a number of methods and programs for analyzing calculated data were developed.
In the future, we are going to adapt the programs for general use and apply them to the Galactica system.

\section{XV.CONCLUSION}

We aimed that the paper contained the necessary information so that even a novice researcher could use Galactica System in his/her works. And there is a great range of problems that can be solved using Galactica. Such studies are within the capacity of one- or two-year students and even senior apt pupils. Within 2-3 years, they can reach a high level and by the time they graduate, they will be able to solve a serious scientific problem and contribute to the understanding of the world.

\section{ACKNOWLEDGMENT}

L. J. Smulsky, Ya. J. Smulsky and O. I. Krotov help me at developing Galactica system at different stages. The calculations with using Galactica were performed on the supercomputers of the Siberian Supercomputing Centre in the ICMMG SB RAS, Novosibirsk, Russia. The reviewer' comments were useful to improve of the paper.

\section{REFERENCES}

[1] J. J. Smulsky, "Galactica Software for Solving Gravitational Interaction Problems," Applied Physics Research, Vol. 4, No. 2, 2012, pp. 110-123. http://dx.doi.org/10.5539/apr.v4n2p110.

[2] J.J. Smulsky, "Axisymmetrical problem of gravitational interaction of N-bodies," Mathematical modeling, Vol. 15, No 5, 2003, pp. 27-36. (In Russian

http://www.smul1.newmail.ru/Russian1/IntSunSyst/ Osvnb4.doc).

[3] I. I. Smul'skii, "Multilayer Ring Structures," Physics of Particles and Nuclei Letters, Vol. 8, No. 5, 2011, pp. 436-440. DOI: 10.1134/S1547477111050189. http://www.ikz.ru/ smulski/Papers/PHPL436.pdf.

[4] J. J. Smulsky, "The Theory of Interaction," Publishing House of Novosibirsk University, Novosibirsk, $1999 . \quad$ (in Russian http://www.ikz.ru/ smulski/TVfulA5 2.pdf).

[5] J. J. Smulsky, "The Theory of Interaction," Publishing house Cultural Information Bank, Ekaterinburg, 2004. (in English http://www.ikz.ru/ smulski/TVEnA5 2.pdf)

[6] J. J. Smulsky, "Dynamics (The skeleton of lectures on the theoretical mechanics for building specialities). The second edition," Tyumen State ArchitectonicBuilding University, Tyumen, Russia, 2004. (in Russian http://www.ikz.ru/ smulski/Dinmk3A5.pdf).

[7] V. P. Melnikov and J. J. Smulsky, "Astronomical Theory of Ice Ages: New Approximations. Solutions and Challenges," Academic Publishing House, Novosibirsk, 2009. http://www.ikz.ru/ smulski/Papers/AsThAnE.pdf.

[8] E. A. Grebenikov and J. J. Smulsky, "Numerical Investigation of the Mars Orbit Evolution in the Time Interval of Hundred Million," A. A. Dorodnitsyn Computing Center of RAS, Moscow, 
$2007 . \quad$ (in Russian http://www.ikz.ru/ smulski/Papers/EvMa100m4t2.p df).

[9] J. J. Smulsky and Ya. J. Smulsky, "Evolution of Apophis Orbit for 1000 Years and New Space Targets," Protecting the Earth Against Collisions with Asteroids and Comet Nuclei, Proceedings of the International Conference "Asteroid-Comet Hazard2009", "Nauka", St.-Petersburg, Russia, 2010. http://www.ikz.ru/ smulski/Papers/EvlAp3Ec.pdf.

[10] J. J. Smulsky and Ya. J. Smulsky, "Dynamic Problems of the Planets and Asteroids, and Their Discussion," International Journal of Astronomy and Astrophysics, Vol. 2, No. 3, 2012, pp. 129-155. doi:10.4236/ijaa.2012.23018.

[11] E. M. Standish, "JPL Planetary and Lunar Ephemerides," Interoffice Memorandum, JPL IOM 312. $F-98-048,1998$.

ftp://ssd.jpl.nasa.gov/pub/eph/planets/ioms/de405.io m.pdf.

[12] V. P. Mel'nikov, I.I. Smul'skii and Ya. I. Smul'skii, "Compound modeling of Earth rotation and possible implications for interaction of continents," Russian Geology and Geophysics, Vol. 49, No. 11, 2008, pp. 851-858. doi:10.1016/j.rgg.2008.04.003.

[13] J. J. Smulsky, "Compound model of rotation of the Sun and displacement of Mercury perihelion," The Fundamental and Applied Problems of the Mechanics: Proceeding of the VI All-Russian scientific Conference, Tomsk State University. Tomsk, Russia, 2008, pp. 433-434 (in Russian http://www.ikz.ru/ smulski/Papers/ModSun51c.pdf).

[14] J. J. Smulsky, "Gravitation, Field and Rotation of Mercury Perihelion," Proceedings of the Natural Philosophy Alliance. 15th Annual Conference, University of New Mexiko, Albuquuerque, USA, Vol. 5, No. 2, 2009, pp. 254-260. http://www.ikz.ru/ smulski/Papers/08Smulsky2c.pdf

[15] J. J. Smulsky, "New Components of the Mercury's Perihelion Precession," Natural Science, Vol. 3, No.4, 2011, pp. 268-274. doi:10.4236/ns.2011.34034. http://www.scirp.org/journal/ns.

[16] J. J. Smulsky, "Computer calculation of components of the Mercury's perihelion precession," Computer Algebra Systems in Teaching and Research. Differential Equations, Dynamical Systems and Celestial Mechanics, Publishig House Collegium Mazovia, Siedlce, Poland, 2011, pp. 217-223. http://www.ikz.ru/ smulski/Papers/ComCMePr2c.pd f.

[17] J. J. Smulsky, "Numerical modeling of evolution of the satellite of a rotating body," The Theoretical and Applied tasks of the Nonlinear Analysis. Russian Academy of Sciences: A.A. Dorodnicyn Computing Center. Moscow, 2008, pp. 100-117. (In Russian http://www.ikz.ru/ smulski/Papers/ModSun07c.pdf).

[18] J. J. Smulsky and Ya. J.Smulsky, "Apophis Movement during 1000 Years and its Possible
Change," Modern Ballistics and Adjacent Questions of the Mechanics: The Collection of Scientific Conference Papers, Tomsk State University, Tomsk, Russia, 2010, pp. 315-316. ( In Russian http://www.ikz.ru/ smulski/Papers/DvizhAp3.pdf).

[19] J. J. Smulsky and Ya. J. Smulsky, "The Transformation of Asteroids in the Satellites - Real Direction in Counteracting Asteroid Hazard of the Earth," Proceeding of the Congress-2010 Fundamental Problems of Natural Sciences and Engineering. Part II $(M-U)$. A Series "Problems of the Universe Researching". Issue 34-2. St.Petersburg, 2010, pp. 263-272. (In Russian http://www.ikz.ru/ smulski/Papers/PrAsSp04c.pdf).

[20] J. J. Smulsky, "Movement of asteroid 1950 DA during 1000 years and possible transformation of asteroid into the satellite," The Fundamental and Applied Problems of the Mechanics: Proceeding of the scientific Conference, University Publishing House, Tomsk, Russia, 2011, pp. 400-401. (In Russian http://www.ikz.ru/ smulski/Papers/Dv1950DA.pdf.

[21] J. J. Smulsky and O. I. Krotov, "Computing of the Apophis's movement during 100 years on two approaches: Galactica and Horisons," The Fundamental and Applied Problems of the Mechanics: Proceeding of the scientific Conference, University Publishing House, Tomsk, Russia, 2011, pp. 402-403. (In Russian http://www.ikz.ru/ smulski/Papers/ClApGlH20.pdf).

[22] California Institute of Technology, "JPL Small-Body Database 2008. Jet Propulsion Laboratory. 99942 Apophis (2004 MN4)," 2008. http://ssd.jpl.nasa.gov/sbdb.cgi?sstr=Apophis;orb=1.

[23]E. Bowell, "The Asteroid Orbital Elements Database," 2008. ftp://ftp.lowell.edu/pub/elgb.

[24] J. J. Smulsky, "Designing of ring structures," The Fundamental and Applied Problems of the Mechanics: Proceeding of the VI All-Russian scientific Conference, University Publishing House, Tomsk, Russia, 2008, pp. 431-432. (In Russian http://www.ikz.ru/ smulski/Papers/CnsKoStr2.pdf).

[25] J. J. Smulsky, "Optimization of Passive Orbit with the Use of Gravity Maneuver," Cosmic Research, Vol. 46, No. 5, 2008, pp. 456-464. http://www.ikz.ru/ smulski/Papers/COSR456.PDF.

Prof. Joseph J. Smulsky is a chief scientist of the Institute of the Earth's Cryosphere of the Siberian Branch of the Russian Academy of Science, doctor of physical and mathematical sciences, professor of theoretical and applied mechanics. He is the author and the co-author more than 150 scientific works and inventions, including four monographs. In research, the interests of Smulsky J.J include mechanics of a liquid and gas, ecology, wind energy, electrodynamics, the theoretical mechanics etc.: http://www.smul1.newmail.ru/. 
Appendix 1. Example of MathCad program for generating an initial condition file.

\section{Iñner.mcd . Example of calculation of the initial conditions for the program Galactici Completed 11.02.2012, modified 05.11.2012. 21:00}

\subsection{Number of bodies}
N 6 - -30
$\mathrm{N}-\mathrm{N} b-1$
i $-0 .$. N
i1 $-1 . .1$
13.2. Constants
G $-6.67259 \cdot 10^{11}$ A U $-1.4959787069110^{11} \quad P_{s d}-365.25636042 \quad k_{s d} J c-\frac{P s d}{36525} \quad d s c-24.3600 \quad P_{s d s c}-P_{s d} d d s c$

13.3. Properties of bodies and of their motions
m E r $-5.9742 \cdot 10^{24}$
M S $-332951.3 \mathrm{~m} \mathrm{E} \mathrm{r}$
M SS $-1.99178735028210^{30}$
Roba -5000
$k_{a}-1.0$
$a-k_{a} \cdot \mathrm{A} U$
e -0.2056
$\varepsilon 0 \quad-0.409$

13.4. Masses of bodies in $\mathrm{kg}$ and theirradiuses in $\mathrm{m}$

${ }^{m} 0-M s \quad m_{i 1}-\frac{M s s-M s}{N} \quad \quad r a m_{i}-\left(\frac{3 \cdot m_{i}}{4 \cdot \pi \cdot R_{0 b a}}\right)^{\frac{1}{3}}$

13.5.Coordinates and velocities of bodies acordindly to:

Smulsky J.J. Axisymmetrical problem of gravitational interaction of N-bodies // Mathematical modelling. - 2003, Vol. 15, Nc 5, Pp. 27-36. (In Russian http://www.smul1 .newmail.ru/Russian 1/IntSunSyst/Osvnb4.doc).
$\alpha_{1}--\frac{1}{1+e}$
$R_{0}-\frac{2 \cdot \alpha_{1}+1}{\alpha_{1}}$
$\mathrm{f}_{\mathrm{N}}-0.25 \cdot \sum_{\mathrm{i} 2=2}^{\mathrm{N}} \frac{1}{\sin \left[\frac{(\mathrm{i} 2-1) \cdot \pi}{\mathrm{N}}\right]}$
$\mu l--G \cdot\left(\mathbb{m}_{0}+\mathrm{m}^{1 \cdot \mathrm{f}} \mathrm{N}\right)$
VD $-\sqrt{\frac{\mu 1}{\alpha_{1} \cdot R D}}$
$d \phi-\frac{2 \cdot \pi}{N} \quad \phi 0_{i 1}-(\mathrm{i} 1-1) \cdot d \phi$
$\alpha_{1}=-0.829462508294625$

Coordinates and velocities in the plane of orbits, in $\mathrm{m}$ and $\mathrm{m} / \mathrm{sec}$

$$
\begin{aligned}
& x_{0} 0_{i 1}-R D \cdot \cos \left(\phi 0_{i 1}\right) \\
& { }^{v 0_{i 1}}-R p \cdot \sin \left(\phi 0_{i i}\right) \\
& { }^{20}{ }_{i 1}-0 \\
& \mu \mathrm{l}=-1.3282469098036210^{20} \\
& v x_{i 1}--v 0 \cdot \sin \left(\phi \theta_{i 1}\right) \\
& v \mathrm{vo}_{i 1}-v D \cdot \cos \left(\phi 0_{i 1}\right) \\
& \mathrm{v}_{\mathrm{r}} \mathrm{il}_{\mathrm{i}}-0 \\
& \mathrm{f}_{\mathrm{N}}=16.12083740509405
\end{aligned}
$$

Checking the distanses between bodies. If $d$ is less than $\mathbf{5 0}$, it is need to increase a.

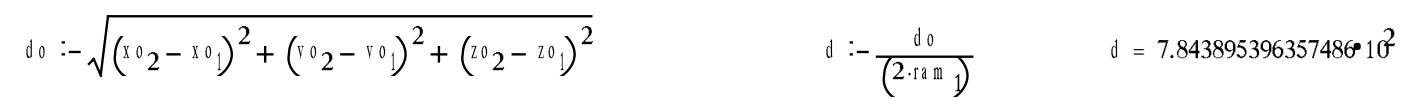

13.6.The exact motion of bodies. Checking the planned configuration of the problem

$$
\begin{aligned}
& \text { J }-50 \quad \phi_{0}-0 \quad i-1 . J \quad \quad \phi_{i}-\phi_{i}-1+\frac{2 \cdot \pi}{J} \quad \mathrm{rnb}_{i 1, i}-\frac{R_{D}}{\left[\left(\alpha_{1}+1\right) \cdot \cos \left(\phi_{i}-\phi 0_{i 1}\right)-\alpha_{1}\right]} \\
& R_{a}-\frac{R_{0}}{\left[-\left(\alpha_{1}+1\right)-\alpha_{1}\right]} \quad D_{M}-1.1 R_{a} \quad x n b_{i 1, i}-\operatorname{rn} b_{i 1, i} \cdot \cos \left(\phi_{i}\right) \quad \quad v n b_{i 1, i}-r n b_{i 1, i} \cdot \sin \left(\phi_{i}\right) \quad i 2-1,3 \ldots N
\end{aligned}
$$

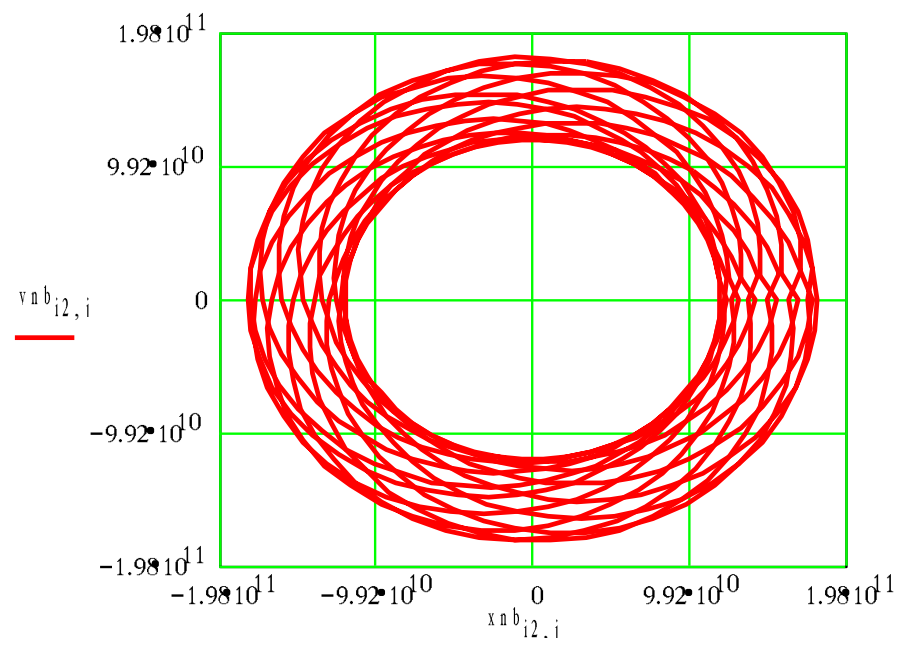




\section{Appendix 1 (continued)}

\begin{tabular}{|c|c|c|}
\hline \multicolumn{3}{|l|}{$\equiv$} \\
\hline- & \multicolumn{2}{|c|}{ 13.7.Coordinates and velocities in the equatorial plane } \\
\hline$x_{i 1}-x_{i 1}$ & $\mathrm{rm} \mathrm{m}_{\mathrm{i} 1}-\mathrm{v0_{i1 }} \cdot \cos (\varepsilon 0)-20_{i 1} \cdot \sin (\varepsilon 0)$ & $2 \mathrm{~m}_{i 1}-v 0_{i 1} \cdot \sin (\varepsilon 0)+20_{i 1} \cdot \cos (\varepsilon 0)$ \\
\hline $\mathrm{rxm} \mathrm{s}_{\mathrm{il}}-\mathrm{vx0_{i1 }}$ & $\nabla \mathrm{rms} s_{i 1}-\nabla v 0_{i 1} \cdot \cos (\varepsilon 0)-\nabla z 0_{i 1} \cdot \sin (\varepsilon 0)$ & $r z m s_{i 1}-r v 0_{i 1} \cdot \sin (\varepsilon 0)+r z 0_{i 1} \cdot \cos (\varepsilon 0$ \\
\hline$x \mathrm{~m} 0_{0}-0$ & $\mathrm{rxms} 0-0$ & $\mathrm{vrms} 0-0$ \\
\hline
\end{tabular}

13.8.The transition to the dimensionless variables

$$
{ }^{m s} s_{0}-0 \quad m s_{i}+1-m s_{i}+m_{i} \quad \quad m o_{i}-\frac{m_{i}}{m s_{N}+1}
$$

Determination of the length scale that the dimensionless time unit is equel one sidereal century

$$
\begin{aligned}
& k_{1}-\frac{1}{100 \mathrm{P} s d s c} \quad A \mathrm{~m}-\left[\frac{\left(G \cdot m s_{N}+1\right)}{k_{f^{2}}^{2}}\right]^{\frac{1}{3}} \quad A \mathrm{~m}=1.09796077030958110^{13} \\
& \text { Clarification of Am }
\end{aligned}
$$

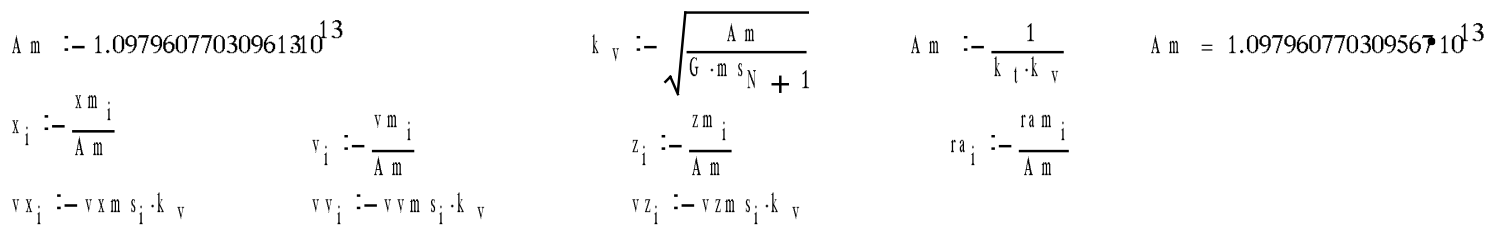

$$
\begin{aligned}
& \text { 13.9. The center of mass of the interacting bodies }
\end{aligned}
$$

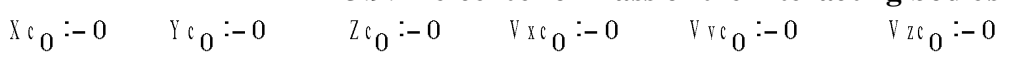

$$
\begin{aligned}
& X c_{i}+1-X c_{i}+\mathbb{m} o_{i} \cdot x_{i} \quad \quad Y c_{i}+1-Y c_{i}+m o_{i} \cdot v_{i} \quad Z c_{i}+1-Z c_{i}+\mathbb{m} o_{i} \cdot z_{i} \\
& V x c_{i}+1-V x c_{i}+m o_{i} \cdot v x_{i} \quad V v c_{i}+1-V v c_{i}+m o_{i} \cdot v v_{i} \quad V z c_{i}+1-V z c_{i}+m o_{i} \cdot v z_{i}
\end{aligned}
$$

Coordinates and velocities relatively the center of mass

$$
\begin{aligned}
& \mathrm{X}_{\mathrm{i}}-\mathrm{X}_{\mathrm{i}}-\mathrm{X}_{\mathrm{C}} \mathrm{N}+1 \quad \mathrm{~V}_{\mathrm{i}}-\mathrm{r}_{\mathrm{i}}-\mathrm{Y} \mathrm{c}_{\mathrm{N}}+1 \quad \mathrm{z}_{\mathrm{i}}-\mathrm{z}_{\mathrm{i}}-\mathrm{Zc}_{\mathrm{N}}+1 \quad \mathrm{X}_{\mathrm{C}} \mathrm{N}+1=0
\end{aligned}
$$

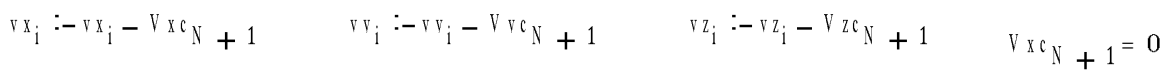

13.10.Angular momentum of the system of bodies

$$
\begin{aligned}
& \mathrm{M} \mathrm{x}_{\mathrm{i}}-0 \quad \mathrm{Mr}_{\mathrm{i}}-0 \quad \mathrm{Mz}_{\mathrm{i}}-0 \\
& M 1 x_{i}--m o_{i} \cdot\left(v v_{i} \cdot z_{i}-v z_{i} \cdot v i\right) \quad M 1 v_{i}-m o_{i} \cdot\left(v x_{i} \cdot z_{i}-v z_{i} \cdot x_{i}\right) \quad M 1 r_{i}-m o_{i} \cdot\left(v v_{i} \cdot x_{i}-v x_{i} \cdot v i\right) \\
& M x_{i}+1-\left(M x_{i}\right)+M 1 x_{i} \quad M v_{i}+1-\left(M r_{i}\right)+M 1 r_{i} \quad M z_{i}+1-\left(M z_{i}\right)+M 1 I_{i}
\end{aligned}
$$

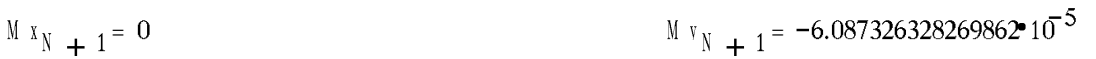

$$
\begin{aligned}
& { }^{M} z_{\mathrm{N}}+1=1.40441278469043910^{-4} \\
& \text { Verification }
\end{aligned}
$$

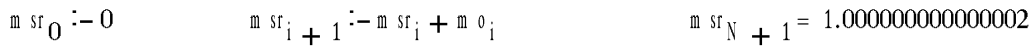

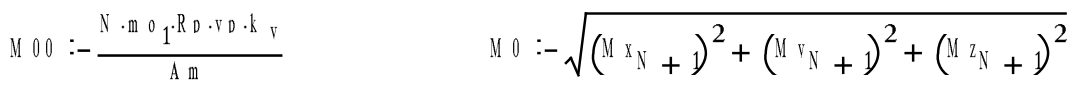

$$
\begin{aligned}
& \frac{M 0-M 00}{M O}=0 \quad\left(A m-\frac{1}{K_{t} \cdot K_{V}}\right) \cdot \frac{1}{A m}=0
\end{aligned}
$$

13.11.To coordinate the size of area with the number of bodies need to change the $B<=1$ and $\mathrm{C} 1<=1$ so that $\mathrm{Nbar}>=$ Nb.

$$
\begin{aligned}
& \text { B }-0.9 \quad \text { C } 1-0.7955 \quad \text { M u }- \text { ceil }\left[(x b+1)^{\frac{1}{3}}\right] \\
& \text { dT }-1 \cdot 10^{6} \quad M u=4 \\
& \text { B C Mu Nb } \\
& \begin{array}{llll}
0.5 & 0.25 \quad 4 \quad 30
\end{array} \\
& \begin{array}{llll}
0.9 & 0.6 \quad 7 & 300
\end{array}
\end{aligned}
$$

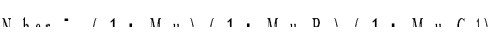




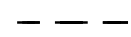

$$
\begin{array}{ll}
k-0 . .14 & 1-7 . .12 \\
R_{0,9}-M_{N}+1 & R_{1,0}-0 \\
R_{1,5}-1 & R_{1,6}-B \\
R_{i}+2,2^{-r_{i}} & R_{i}+2,3-z_{i} \\
R_{i+2,13-a_{i}} & R_{i}+2,14-0
\end{array}
$$

$R_{13,0}-20120917$

$$
R_{13,1}-N b
$$

WW W W RITE PRA"axsvmb30.prn" ) = R
Appendix 1 (continued)

\subsection{Writing data to the file name.prn}

It is necessary 6 ze ro at the end of 2 -nd line of a file name.prn to remove and to rename file into a name.dat.

Verification coordinates and velocities
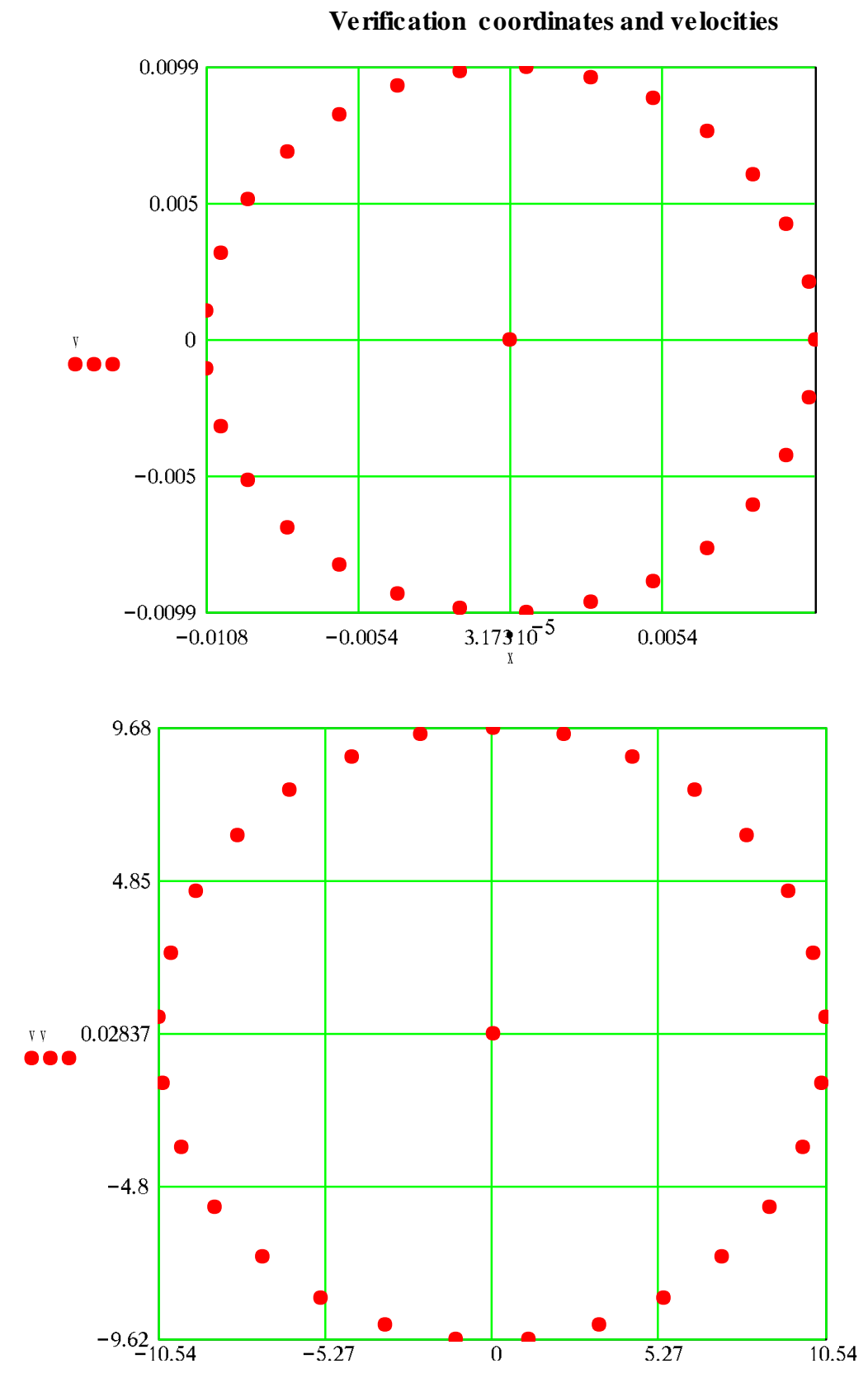

${ }^{R} 0,8-M{ }^{N} N+1$

$\mathrm{R}_{1,4}-\mathrm{Nb}$

$R_{i}+2,1-x_{i}$

$R_{i+2,1}-0$
$\mathrm{R}_{\mathrm{i}}+2,0-\mathrm{m} 0_{\mathrm{i}}$

$R_{i}+2,6-v z_{i}$

$13-N b+2$

Information line at end of file

$R_{13,4}-k_{1}$

$R_{13,12}-0$

"axsvmb3t. prn"

1.5 
Appendix 1 (end)

SWI $\bar{z}=-2.489920288495653 \cdot 10^{-4}$

L ther $-\frac{\text { Yearo }}{d T}$

$L+k \cdot 2=9.995788296740868^{\circ} 10^{3}$

$r_{i 1}-\sqrt{\left(x_{i 1}\right)^{2}+\left(v_{i 1}\right)^{2}+\left(z_{i 1}\right)^{2}}$

$\mathrm{drO}^{\mathrm{i} 1}-\mathrm{r}_{\mathrm{i} 1}-\mathrm{r} \mathrm{O}_{1}$

$v 0_{i 1}-\sqrt{\left(v_{i}\right)^{2}+\left(v_{i 1}\right)^{2}+\left(v_{i}\right)^{2}}$

$\mathrm{dv} \mathrm{O}_{\mathrm{i} 1}-\mathrm{v} \mathrm{O}_{\mathrm{i} 1}-\mathrm{v} \mathrm{O}_{1}$
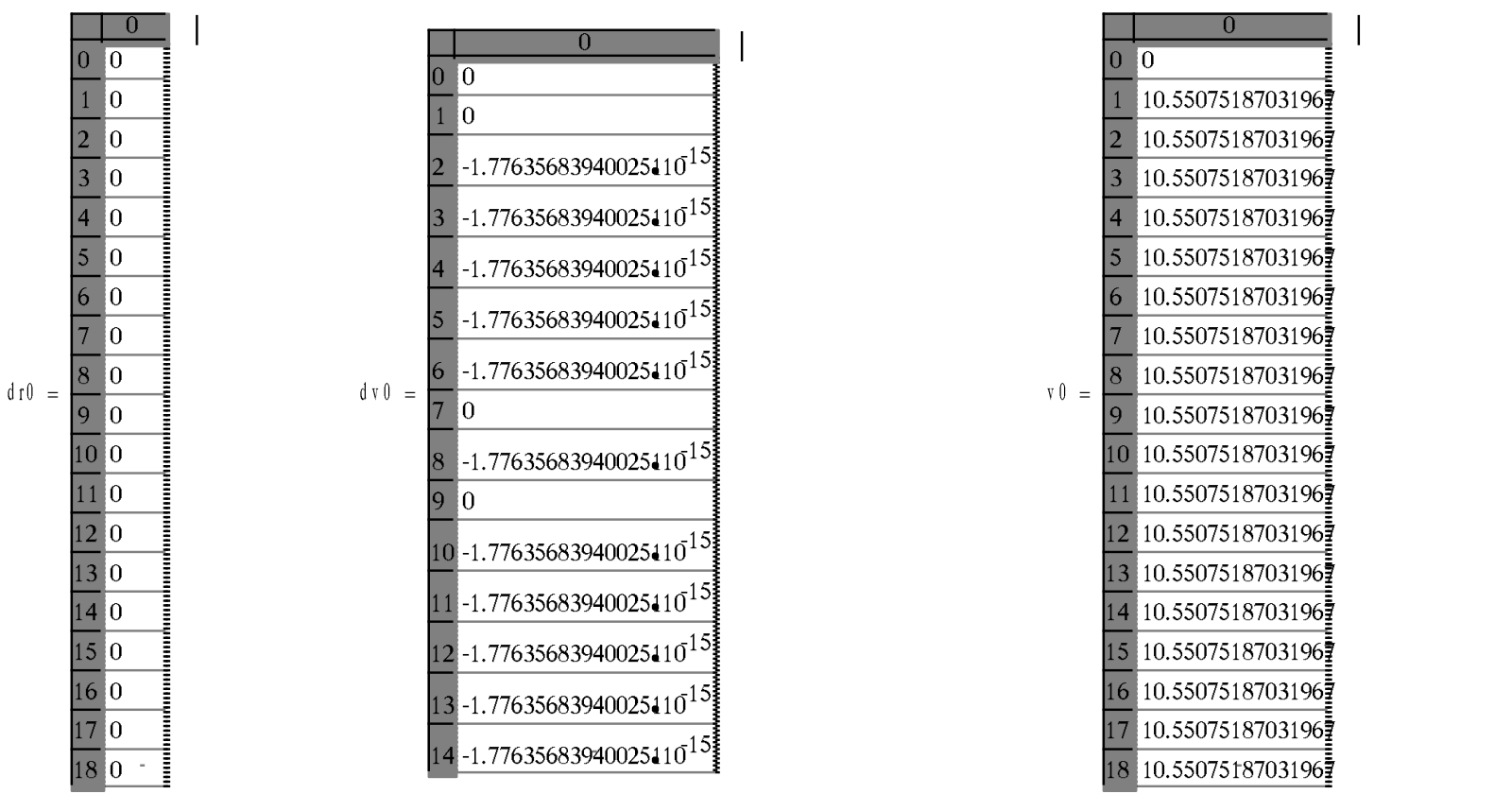

ro $0_{i 1}-\sqrt{\left(x q_{i 1}\right)^{2}+\left({ }^{2} g_{i 1}\right)^{2}+\left(2 g_{i i}\right)^{2}}$

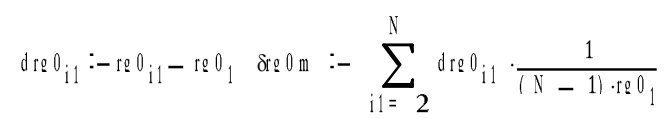

$\delta \mathrm{re} 0 \mathrm{~m}=6.10592511566260110^{-4}$

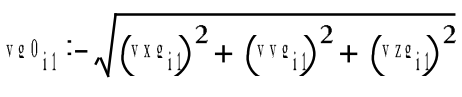

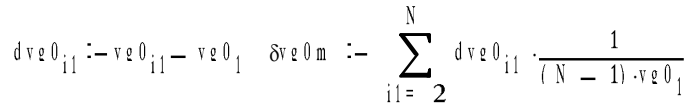

$\delta$ r 0 om $=7.46665157181493610^{-4}$

Verification: errors for $\mathrm{Nb}=300$ in dependance of revolution, $\mathrm{dT}=10^{\wedge}-6$.

re vl

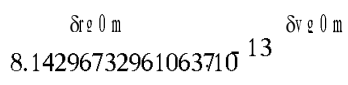

$-1.1321687060818780^{13}$

2

$9.8932843931391810^{10}$

$3.3904762960110471 \sigma^{10}$

3

$8.6649110308178910^{7}$

$6.257378445795779 \cdot 1 \sigma^{7}$

$5.960998639339029 \cdot 10^{4}$

4

$7.23045195903398610^{4}$ 


\section{Appendix 2. Modes of display in the standard graphics of FORTRAN}

\begin{tabular}{|l|l|l|}
\hline Symbolical argument & \multicolumn{1}{|c|}{ Cod } & \multicolumn{1}{c|}{ Description } \\
\hline \$MAXRESMODE & -3 & graphics mode with highest resolution \\
\hline \$MAXCOLORMODE & -2 & graphics mode with most colors \\
\hline \$DEFAULTMODE & -1 & restore screen to original mode \\
\hline \$TEXTBW40 & 0 & $40 \times 25$ text, 16 grey \\
\hline \$TEXTC40 & 1 & $40 \times 25$ text, $16 / 8$ color \\
\hline \$TEXTBW80 & 2 & $80 \times 25$ text, 16 grey \\
\hline \$TEXTC80 & 3 & $80 \times 25$ text, $16 / 8$ color \\
\hline \$MRES4COLOR & 4 & $320 \times 200,4$ color \\
\hline \$MRESNOCOLOR & 5 & $320 \times 200,4$ grey \\
\hline \$HRESBW & 6 & $640 \times 200$, BW \\
\hline \$TEXTMONO & 7 & $80 \times 25$ text, BW \\
\hline \$HERCMONO & 8 & $720 \times 348$, BW for HGC \\
\hline \$MRES16COLOR & 13 & $320 \times 200,16$ color \\
\hline \$HRES16COLOR & 14 & $640 \times 200,16$ color \\
\hline \$ERESNOCOLOR & 15 & $640 \times 350$, BW \\
\hline \$ERESCOLOR & 16 & $640 \times 350,4$ or 16 color \\
\hline \$VRES2COLOR & 17 & $640 \times 480$, BW \\
\hline \$VRES16COLOR & 18 & $640 \times 480,16$ color \\
\hline \$MRES256COLOR & 19 & $320 \times 200,256$ color \\
\hline \$ORESCOLOR & 64 & $640 \times 400,1$ of 16 colors $($ Olivetti) \\
\hline
\end{tabular}

